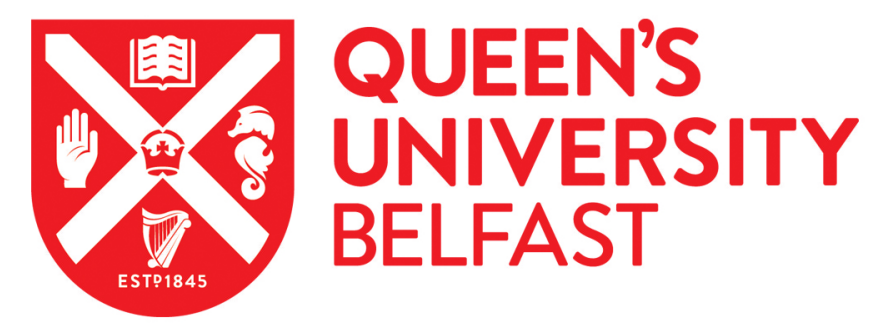

\title{
NLRP3 inflammasome priming: A riddle wrapped in a mystery inside an enigma
}

McKee, C. M., \& Coll, R. C. (2020). NLRP3 inflammasome priming: A riddle wrapped in a mystery inside an enigma. Journal of Leukocyte Biology. https://doi.org/10.1002/JLB.3MR0720-513R

Published in:

Journal of Leukocyte Biology

Document Version:

Peer reviewed version

Queen's University Belfast - Research Portal:

Link to publication record in Queen's University Belfast Research Portal

Publisher rights

(C) 2020 by the Society for Leukocyte Biology.

This work is made available online in accordance with the publisher's policies. Please refer to any applicable terms of use of the publisher.

\section{General rights}

Copyright for the publications made accessible via the Queen's University Belfast Research Portal is retained by the author(s) and / or other copyright owners and it is a condition of accessing these publications that users recognise and abide by the legal requirements associated with these rights.

Take down policy

The Research Portal is Queen's institutional repository that provides access to Queen's research output. Every effort has been made to ensure that content in the Research Portal does not infringe any person's rights, or applicable UK laws. If you discover content in the Research Portal that you believe breaches copyright or violates any law, please contact openaccess@qub.ac.uk. 


\section{Special Section of Journal of Leukocyte Biology \\ 14th World Congress on Inflammation meeting}

Review Title:

NLRP3 inflammasome priming: a riddle wrapped in a mystery inside an enigma

Authors:

Chloe M. McKee ${ }^{1}$ and Rebecca C. Coll

\section{Affiliations:}

${ }^{1}$ The Wellcome-Wolfson Institute for Experimental Medicine, Queen's University Belfast, UK

Correspondence:

Dr Rebecca C. Coll, Wellcome-Wolfson Institute for Experimental

Medicine, Queen's University Belfast, 97 Lisburn Road, Belfast, BT9 7BL, UK. Email: r.coll@qub.ac.uk 


\section{Abbreviations}

AIM2; absent in melanoma 2

ARIH2; Ariadne homolog 2

ASC; apoptosis-associated speck like protein containing a caspase recruitment domain

Bcl-2; B-cell lymphoma 2

BRCC3; BRCA2 containing complex subunit 3

BTK; Bruton's tyrosine kinase

cAMP; cyclic adenosine monophosphate

CAPS; cryopyrin associated periodic syndromes

CARD; caspase recruitment domain

clAP; cellular inhibitor of apoptosis protein

COP; CARD-only protein

CPPD crystals; calcium pyrophosphate dihydrate crystals

CRISPR; clustered regularly interspaced short palindromic repeats

DUB; deubiquitinating enzyme

FBXL2; F- box/LRR- repeat protein 2

FBXO3; F- box only protein 3

GSDMD; gasdermin D

GSTO1-1; glutathione transferase omega-1

HSV-1; Herpes simplex virus type 1

ICE; interleukin-1 beta converting enzyme

IFI16; Interferon-y Inducible Protein 16

IFN; interferon 
IKK; IKB kinase

IKKi; IKK-related kinase

IL; interleukin

Inca; inhibitory CARD

IRAK-1; IL-1 receptor-associated kinase

JNK-1; c-Jun N-terminal kinase 1

LPS; lipopolysaccharide

LRR; leucine rich repeat

LUBAC; linear ubiquitin assembly complex

MARCH7; membrane-associated RING finger protein 7

MAVS; mitochondrial activation signalling protein

MDS; myelodysplastic syndrome

NEK7; NIMA-related kinase 7

NLRP3; NOD-, LRR- and pyrin domain-containing protein 3

PAK1; PI-3K/Rac1/p21-activated kinase 1

PKA; protein kinase $A$

PKD; protein kinase D

PLK4; polo-like kinase 4

POP; PYD-only protein

PP2A; protein phosphatase $2 \mathrm{~A}$

PPI; protein-protein interactions

PRR; pattern recognition receptor

PTM; post-translational modification

PTPases; protein tyrosine phosphatases

PTPN22; protein tyrosine phosphatase non-receptor type 22 
PYD; pyrin domain

Pyk2; proline-rich tyrosine kinase 2

RIP2; receptor-interacting-serine/threonine-protein kinase 2

ROS; reactive oxygen species

SENP; SUMO-specific protease

Ser; serine

SIRT2; sirtuin 2

STING; stimulator of interferon genes

SUMO; small ubiquitin-like modifier

Syk; spleen tyrosine kinase

TGN; trans-Golgi network

TLR; toll-like receptor

TNF; tumour necrosis factor

TRAF; TNFR associated factor

TRIM31; tripartite motif containing protein 31

Tyr; tyrosine

USP; ubiquitin specific peptidase

\section{Abstract}


The NLRP3 (NOD-, LRR- and pyrin domain-containing protein 3) inflammasome is an immunological sensor that detects a wide range of microbial- and host-derived signals. Inflammasome activation results in the release of the potent proinflammatory cytokines interleukin (IL)-1 $\beta$ and IL-18 and triggers a form of inflammatory cell death known as pyroptosis. Excessive NLRP3 activity is associated with the pathogenesis of a wide range of inflammatory diseases, thus NLRP3 activation mechanisms are an area of intensive research. NLRP3 inflammasome activation is a tightly regulated process that requires both priming and activation signals. In particular, recent research has highlighted the highly complex nature of the priming step, which involves transcriptional and post-translational mechanisms, and numerous protein binding partners. This review will describe the current understanding of NLRP3 priming and will discuss the potential opportunities for targeting this process therapeutically to treat NLRP3-associated diseases.

\section{Introduction}


The cytoplasmic innate immune receptor NLRP3 forms a proinflammatory signalling complex known as an inflammasome ${ }^{1}$. NLRP3 has been the subject of intense research since mutations in the NLRP3 gene were first linked to the autoinflammatory Cryopyrin Associated Periodic Syndromes (CAPS) in $2001^{2}$. NLRP3 forms an inflammasome by oligomerising and interacting with the adapter apoptosis-associated speck like protein containing a caspase recruitment domain (ASC) $)^{1,3,4}$. ASC has remarkable prion-like properties and forms large fibrillar aggregations known as 'specks'3,4. These ASC specks provide a platform for the activation of the zymogen caspase- $1^{5}$. Active caspase- 1 cleaves substrates such as the cytokines pro-IL-1 $\beta$, pro-IL-18, and the pore forming protein gasdermin-D (GSDMD) which cause inflammation and cell death (pyroptosis) ${ }^{3-5}$ (Figure 1). It is now apparent that excessive or aberrant activation of NLRP3 can cause damaging inflammation that is associated with the pathogenesis of a huge range of diseases $^{6}$. Despite the significant progress towards understanding the role of NLRP3 in disease and the promising therapeutic potential of modulating its activity, the molecular mechanisms governing NLRP3 activation remain incompletely understood 3 .

NLRP3 can be activated by an astounding variety of microbial-, host-, and environmental-derived molecules ranging from pore forming toxins to monosodium urate crystals and asbestos particles ${ }^{1,4}$. The indiscriminate nature of NLRP3 activation has made defining this mechanism a challenging task for the field, however some key cellular processes involved have been defined. Different types of NLRP3 activators trigger different activation pathways. For example, particulates disrupt lysosomes while certain stimuli cause mitochondrial damage which appears to be necessary for NLRP3 activation ${ }^{4}$. Most of these pathways converge as they all 
ultimately cause potassium efflux from the cell. This change in potassium concentration activates NLRP3, although how NLRP3 senses this change is not known $^{3,7}$. More recently, non-potassium efflux dependent mechanisms of NLRP3 have been defined. They involve the disruption of the mitochondrial electron transport chain ${ }^{8}$ or a TLR4-TRIF-RIPK1-FADD-Caspase-8 signalling pathway present in human monocytes termed alternative NLRP3 activation ${ }^{9}$. In addition, a mechanism triggered by both potassium-dependent and -independent NLRP3 stimuli is the disassembly of the trans-Golgi network (TGN) which facilitates NLRP3 inflammasome assembly ${ }^{10}$. The molecular details of NLRP3 activation signals have been extensively reviewed ${ }^{3,4,11}$ and are not the focus of the discussion herein. In addition to an activating signal, NLRP3 critically requires a stimulus that has become known as a 'priming' or 'licencing' signal in order to become fully active. The priming of the NLRP3 inflammasome is a multifaceted process involving transcriptional and post-translational mechanisms and the regulation of protein partners that interact with NLRP3 ${ }^{11,12}$.

Unfortunately, the use of the term 'priming' and the specific cellular events it refers to has been inconsistent and is confused in the literature. To prime something means to make it ready or prepare it for use. Indeed, the earliest use of the verb in the 16th century describes the action to fill or load a weapon ${ }^{13}$. The use of priming in reference to NLRP3 is thus entirely apt as the NLRP3 inflammasome is a piece of cellular artillery with the ability to initiate powerful inflammation and cell destruction. NLRP3 activation is therefore strictly controlled and priming is a key component of the security measures that ensure a timely and appropriate inflammatory response. This review will attempt to demystify NLRP3 priming and will describe the current 
understanding of this enigmatic process and the potential for its therapeutic modulation.

\section{The Two-step Activation Model}

Prior to the description of the inflammasome ${ }^{14}$ and the demonstration that NLRP3 could form such a complex in $2004^{15}$ many studies had focused on the posttranslational processing of the pro-inflammatory cytokine IL-1 $\beta$. IL- $1 \beta$ is synthesised as an inactive pro-form and must be cleaved or processed into its $17 \mathrm{kDa}$ mature form that can activate signalling through the $\mathrm{IL}-1$ receptor complex ${ }^{16}$. Although the exact nature of the protease responsible for IL-1 $\beta$ processing was initially unclear, a number of studies established that two signals were required to cause pro-IL-1 $\beta$ production and mature IL-1 $\beta$ release ${ }^{17,18}$. The synthesis of pro-IL-1 $1 \beta$ can be induced by many stimuli including Toll-like receptor (TLR) ligands and cytokines such as tumour necrosis factor (TNF)- $\alpha^{16}$. Lipopolysaccharide (LPS) stimulated signalling via TLR4 was and remains the most commonly used ligand to induce pro-IL-1 $\beta$ production due to its potency and ready availability. Studies including those by Christopher Gabel and colleagues identified a number of different signals such as the pore forming toxin nigericin ${ }^{17}$ and potassium depletion ${ }^{19}$ that could trigger the processing of pro-IL-1 $\beta$. Subsequent research demonstrated that these stimuli activate NLRP $3^{7,20}$ and that the enzyme responsible for IL-1 $\beta$ processing is caspase1 (initially know as IL-1 $\beta$ converting enzyme or ICE) ${ }^{21}$. The concept of the two-step activation model of NLRP3 thus originated from previous research on the posttranslational processing of IL-1 $\beta$.

\section{Transcriptional Priming}


As described above, one aspect of the NLRP3 inflammasome priming process is the

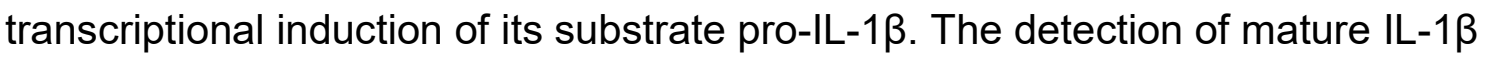

continues to be a key experimental read-out in many inflammasome studies. Another important inflammasome substrate is pro-IL-18. Pro-IL-18 is constitutively expressed in numerous cell types such monocytes and epithelial cells. However, pro-IL-18 expression can also be induced by TLR signalling ${ }^{22}$, thus the relative importance of its transcriptional regulation is context dependent.

NLRP3 itself is also regulated at the transcriptional level. Although NLRP3 is present in unstimulated cells, TLR and cytokine stimulation significantly increase its expression ${ }^{23,24}$ by activating the transcription factor NF-KB ${ }^{24}$. Bauernfeind et al. (2009) demonstrated that ectopic constitutive expression of NLRP3 in macrophage cell lines rendered them sensitive to NLRP3 activation in the absence of a priming signal $^{24}$. These results suggest that high levels of NLRP3 expression are sufficient to prime the NLRP3 inflammasome for activation. Whether such high levels of NLRP3 expression are reached physiologically is unclear, but enhanced NLRP3 expression appears to correlate with higher levels of inflammasome activation in macrophages ${ }^{25}$. The second facet of transcriptional NLRP3 inflammasome priming is thus the induction of NLRP3, as illustrated in Figure 1.

\section{Post-translational Priming}

Although transcriptional priming may enhance NLRP3 activation and is required for $\mathrm{IL}-1 \beta$ production, more recent studies have demonstrated that transcription is not necessary for NLRP3 activation. Numerous studies have reported that simultaneous addition of priming and activation stimuli can activate NLRP3 ${ }^{25-29}$. As it takes around 
two hours to upregulate NLRP3 protein expression ${ }^{24}$, post-translational processes are believed to account for this rapid activation of NLRP $3^{30}$. Analysis of the signalling pathways downstream of priming by TLRs has observed that the adapter molecule MyD88 and the IL-1 receptor-associated kinases IRAK-1 and IRAK-4 are essential for fast NLRP3 priming ${ }^{28,29}$. LPS sensed by TLR4 rapidly activates IRAK1 and IRAK4 via MyD88 and so priming of NLRP3 within minutes is consistent with known TLR responses ${ }^{31}$. Importantly, pharmacological inhibitors of NF-kB and of protein synthesis fail to block rapid NLRP3 activation ${ }^{29,32}$ suggesting that post-translational modifications (PTMs) are the essential regulators of NLRP3 activation.

\section{Post-translational Modifications}

PTMs influence many aspects of protein function including their activity, degradation, localisation, structure, and interactions with other proteins; PTMs are thus essential for rapid protein regulation ${ }^{33,34}$.

\section{Ubiquitination of NLRP3}

In the first study identifying a role for PTMs in regulating NLRP3, Juliana et al. (2012) demonstrated that signalling through TLR4 and MyD88 triggers deubiquitination of NLRP3 to induce inflammasome activation ${ }^{26}$. Additional studies have confirmed that deubiquitinating enzymes (DUBs) play a vital role in NLRP3 regulation as small molecule inhibitors of DUBs completely block NLRP3 activation ${ }^{26,35,36}$. Deubiquitination of the leucine rich repeat (LRR) domain of NLRP3 by BRCA2 containing complex subunit 3 (BRCC3, human BRCC36) is necessary for NLRP3 oligomerisation ${ }^{36}$. LPS stimulation induces ABRO1, a subunit of the BRCC3 deubiquitinase complex, to bind to NLRP3 and recruit BRCC3. Upon subsequent 
activation signals, BRCC3 removes K63-linked ubiquitin from NLRP3, allowing inflammasome activation ${ }^{37}$. In addition, the stimulator of interferon genes (STING) has been shown, upon Herpes simplex virus type 1 (HSV-1) infection and cytosolic DNA stimulation, to bind to NLRP3 and localise it to the endoplasmic reticulum, where it then removes K48- and K63-linked polyubiquitination of NLRP3, facilitating inflammasome activation ${ }^{38}$. Additional DUBs, such as ubiquitin-specific peptidase 7 (USP7) \& USP47, have also been shown to play an essential role in regulating inflammasome activation, although they are activated primarily in response to NLRP3 activation signals like nigericin and calcium pyrophosphate dihydrate (CPPD) crystals ${ }^{39}$.

Ubiquitination also negatively regulates NLRP3 by inducing protein degradation. Fbox/LRR- repeat protein 2 (FBXL2) ubiquitinates the LRR domain of NLRP3 to promote its proteasomal degradation in resting cells ${ }^{40}$. This process is inhibited following LPS stimulation as the E3 ligase component, F-box only protein (FBXO3) targets and degrades FBXL2, thereby extending the half-life of NLRP3 ${ }^{40}$. The E3 ligases tripartite motif containing protein 31 (TRIM31) and Ariadne homolog 2 (ARIH2) bind to the NLRP3 pyrin (PYD) and NACHT domains respectively to induce K48-linked ubiquitination and subsequent proteasome-mediated degradation of NLRP3 ${ }^{41,42}$. Stimulation with LPS further increases the expression and E3 ligase activity of TRIM31, suggesting it functions as a feedback suppressor of inflammasome activation ${ }^{41}$. However, a more recent report from Tang et al. (2020), found that TRIM31 does not interact with NLRP3, suggesting it may not be the E3 ligase that targets NLRP3 for ubiquitination ${ }^{43}$. These contradictory findings could be due to differences in experimental approach as Song et al. (2016) examined cells from TRIM31-- mice while Tang et al. (2020) use siRNA to knockdown TRIM31. In 
addition, different TRIM31 antibodies were used to study NLRP3-TRIM31 interactions ${ }^{41,43}$. Tang et al. (2020) instead identified sequential ubiquitination of NLRP3 by the E3 ligases RNF125 and Cbl-b as critical for proteasomal degradation of NLRP3. RNF125 first induces K63-linked ubiquitination of the LRR domain of NLRP3, recruiting Cbl-b which induces K48-linked polyubiquitination of NLRP3 at K496, thus targeting NLRP3 for degradation ${ }^{43}$. In addition, dopamine signalling through dopamine receptor D1, triggers an increase in cyclic (c)AMP, promoting K48-linked ubiquitination of the NLRP3 LRR domain by the E3 ubiquitin ligase membrane-associated RING finger protein 7 (MARCH7) and inducing NLRP3 degradation via autophagy ${ }^{44}$.

Additional E3 ligases, such as Cullin1, a component of the Skp1-Cullin1-F-box E3 ligase, inactivate, but do not degrade NLRP3 ${ }^{45}$. In resting cells and following LPS stimulation, Cullin1 interacts with NLRP3 and promotes NLRP3 ubiquitination, which represses inflammasome activation without causing protein degradation. Only following the addition of a second activating signal (nigericin, ATP or influenza A virus H3N2) does Cullin1 dissociate from NLRP3 and allow inflammasome formation ${ }^{45}$.

More recently, studies have found that ubiquitination also positively regulates NLRP3 activation. The E3 ubiquitin ligase Pellino2 facilitates NLRP3 activation by triggering K63-linked ubiquitination of NLRP3 during LPS priming ${ }^{46}$. However, NLRP3 has not been shown to be a direct target of Pellino2, suggesting that ubiquitination may be mediated by an additional E3 ubiquitin ligase. Although the molecular mechanisms are not yet clear, ubiquitination mediated by Pellino2 may prevent inhibitory proteins, such as IRAK1, from binding to NLRP346. Presumably, once this ubiquitin has performed its role in the priming process, the ubiquitin chains need to be removed by 
BRCC3 to allow inflammasome formation ${ }^{46}$. As summarised in Figure 2, multiple E3 ubiquitin ligases and DUBs must co-operate to regulate NLRP3 inflammasome activation. Priming (generally LPS stimulation) triggers many of these changes in NLRP3 ubiquitination, demonstrating that ubiquitination is a key component of the NLRP3 priming process.

\section{Phosphorylation of NLRP3}

Phosphorylation is another major PTM that regulates NLRP3 activity and is essential to the priming process. During priming, c-Jun N-terminal kinase 1 (JNK1) phosphorylates NLRP3 at human Serine 198 (Ser198, mouse Ser194) which is critical for NLRP3 self-association and inflammasome activation ${ }^{30}$. Phosphorylated Ser198 is present 15 minutes after treatment with LPS, demonstrating that phosphorylation occurs early in the priming process and can partly account for rapid priming observed through non-transcriptional mechanisms ${ }^{26,30}$. LPS priming and subsequent NLRP3 activating signals also trigger activation of protein kinase D (PKD) which phosphorylates human Ser295 (mouse Ser293) of NLRP3 at the Golgi membrane, releasing NLRP3 to allow formation of the inflammasome ${ }^{47}$. However, phosphorylation at Ser295 is also inhibitory. Mortimer et al. (2016) demonstrated that protein kinase A (PKA) directly phosphorylates Ser295 in NLRP3, inhibiting the ATPase function of the NACHT domain, which is necessary for NLRP3 oligomerisation ${ }^{48}$. Future studies are needed to elucidate why phosphorylation of the same serine residue by two different kinases has opposing effects.

Other inhibitory phosphorylation events have been identified on NLRP3. Phosphorylation at human Ser5 (mouse Ser3) inhibits NLRP3 inflammasome activation as Ser5 is located at a PYD-PYD interface and phosphorylation interferes 
with these charge-charge interactions ${ }^{49}$. Ser5 must be dephosphorylated by protein phosphatase 2A (PP2A) to allow inflammasome activation following additional activation signals ${ }^{49}$. This activity of PP2A is inhibited by Bruton's tyrosine kinase (BTK) which binds to NLRP3 following LPS stimulation to prevent dephosphorylation of Ser5. This inhibition is relieved upon NLRP3 activation which triggers the dissociation of BTK and NLRP350. Phosphorylation at Tyrosine 861 (Tyr861) of NLRP3 is also inhibitory and in resting cells prevents its aberrant activation. Tyr861 is dephosphorylated by protein tyrosine phosphatase non-receptor type 22 (PTPN22) in response to NLRP3 activation stimuli to allow inflammasome assembly ${ }^{51}$. It appears that Ty861 phosphorylation drives NLRP3 into autophagosomes consequently limiting its activation ${ }^{52}$. Whether Tyr861 phosphorylation is affected by priming stimuli is unclear. Recently, Huang et al. (2020) demonstrated that dephosphorylation of NLRP3 at Tyr32 by the phosphatase PTEN is necessary to allow NLRP3 activation and subsequent anti-tumour activity in response to chemotherapy, highlighting the potential clinical importance of NLRP3 $\mathrm{PTMs}^{53}$. As illustrated in Fig 2, the activities of numerous kinases and phosphatases must be co-ordinated to enable both NLRP3 priming and activation.

\section{Additional PTMs of NLRP3}

Evidence in recent years has highlighted a role for additional PTMs in regulating NLRP3 (Figure 2). NLRP3 is sumoylated by the small ubiquitin-like modifier (SUMO) E3 ligase MAPL in resting cells and is desumoylated by the SUMO-specific protease 6 (SENP6) and SENP7 in response to NLRP3 activation signals ${ }^{54}$. However, sumoylation can also be activating, as sumoylation by SUMO1 is necessary for NLRP3 activation and desumoylation mediated through SENP3 attenuates 
activation $^{55}$. Importantly, LPS priming does not appear to affect NLRP3 sumoylation ${ }^{54,55}$.

Multiple studies have identified nitrosylation as a negative regulator of NLRP3 ${ }^{56-58}$. Mycobacterium tuberculosis infection, pre-treatment with interferon (IFN)- $\beta$, or longterm LPS stimulation, trigger IFN- $\gamma$-mediated S-nitrosylation of NLRP3 which inhibits inflammasome assembly ${ }^{56,58}$. It has also been demonstrated that endogenous nitric oxide negatively regulates NLRP3 through stabilising mitochondria ${ }^{57}$.

Recently He et al. (2020) identified an 'acetylation switch' that is involved in the regulation of the NLRP3 inflammasome. They demonstrate that NLRP3 acetylation at $\mathrm{K} 21$ and $\mathrm{K} 22$ in macrophages is necessary for inflammasome activation, while the cytosolic deacetylase sirtuin 2 (SIRT2) inhibits activation ${ }^{59}$. A preprint study from Zhao et al. (2019), has observed that this acetylation is triggered specifically by the second NLRP3 activation signal, not a priming signal, which is mediated by KAT5 and regulates NLRP3 aggregation and association with $\mathrm{ASC}^{60}$.

Other less well characterised PTMs of NLRP3 include alkylation which regulates inflammasome activation through preventing the ATPase activity of NLRP3 ${ }^{61}$ and ADP-ribosylation which is essential for NLRP3 activation following infection with mycoplasma ${ }^{62}$. While only nitrosylation of NLRP3 is clearly influenced by priming stimuli, future studies will likely uncover additional PTMs that play a role in NLRP3 priming.

\section{PTMs of ASC}

In addition to direct regulation of NLRP3, priming also indirectly regulates NLRP3 through PTMs of other components of the inflammasome that are essential to its activity (Figure 3). For example, various ubiquitination and deubiquitination events 
have been shown to be essential for ASC oligomerisation and formation of the NLRP3 inflammasome. The DUB USP50 deubiquitinates ASC through the removal of K63-linked polyubiquitin chains to allow inflammasome formation ${ }^{63}$. Signalling through mitochondrial activation signalling protein (MAVS) triggers TNFR-associated factor 3 (TRAF3)-mediated ubiquitination of $\mathrm{ASC}^{64}$ and the linear ubiquitination assembly complex (LUBAC) mediates linear ubiquitination of $\mathrm{ASC}^{65}$, both of which are essential for NLRP3 inflammasome assembly. However, ASC ubiquitination appears to be regulated by the second activation signal ${ }^{63-65}$. ASC phosphorylation also regulates NLRP3 inflammasome activation. Phosphorylation by the IkB kinase (IKK) complex can both positively and negatively regulate ASC. IKKa induces phosphorylation at Ser16 and Ser193 to sequester ASC in the nucleus in resting cells, whereas LPS stimulation promotes IKK-related kinase (IKKi)-driven translocation of ASC to the perinuclear region through the phosphorylation of Ser58 to allow inflammasome formation ${ }^{66}$. ASC is phosphorylated at multiple tyrosine residues, including Tyr146 (located in the caspase recruitment domain, CARD), which is essential for ASC speck formation and caspase- 1 activation ${ }^{67,68}$. Spleen tyrosine kinase (Syk) phosphorylates proline-rich tyrosine kinase 2 (Pyk2) to induce subsequent phosphorylation of ASC at Tyr146 upon activation of both the NLRP3 and Absent in Melanoma 2 (AIM2) inflammasomes ${ }^{68,69}$. Dephosphorylation of ASC by protein tyrosine phosphatases (PTPases) at Tyr60 and Tyr137 is also essential for ASC speck formation and NLRP3 inflammasome activation, highlighting the dynamic nature of PTM-mediated regulation ${ }^{70}$. It is unclear whether phosphorylation of Tyr146 or dephosphorylation of Tyr60 and Tyr137 are directly related to priming.

\section{PTMs of Caspase-1 and pro-IL-1 $\beta$}


Although they do not directly interact with NLRP3, caspase-1 and pro-IL-1 $\beta$ are also regulated by PTMs which can impact the activity of NLRP3, as illustrated in Figure 3. Polyubiquitination of pro-IL-1 $\beta$ is necessary to allow its proteolytic processing via caspase-1 ${ }^{71}$. A20 prevents this K63-linked ubiquitination of pro-IL-1 $\beta$ to prevent spontaneous IL-1 $\beta$ release in response to LPS alone ${ }^{71}$. Also, LPS-induced K63linked polyubiquitination of caspase-1 by cellular inhibitor of apoptosis protein 1 (cIAP1) and clAP2 is required for caspase-1 activity ${ }^{72}$. E3 ligases, including TRAF6 have been shown to play a positive regulatory role in non-transcriptional priming of the NLRP3 inflammasome, although it is not known which components of the inflammasome are targeted ${ }^{73}$. Ubiquitination can also be inhibitory as linear ubiquitination of the CARD of caspase-1 by LUBAC suppresses caspase-1 activation, however it is not clear if this is directly linked to priming ${ }^{74}$. The ability of PTMs to regulate inflammasome activation has also been exploited by pathogens, for example, the bacterial toxin Streptolysin $\mathrm{O}$ induces ubiquitination and degradation of pro-IL-1 $\beta$, therefore playing an important role in bacterial virulence and pathogenesis by limiting availability of pro-IL-1 $\beta$ and subsequently reducing the amount of mature $\mathrm{IL}-1 \beta$ released from macrophages in response to infection ${ }^{75}$. In another example, stimulation of THP-1 cells with Helicobacter pylori LPS triggers activation of PI-3K/Rac1/p21-activated kinase 1 (PAK1). PAK1 phosphorylates caspase-1 Ser376, which appears to be necessary for caspase-1 activation in this context $^{76}$. Whether LPS from other pathogens can trigger caspase-1 phosphorylation is not known. It is apparent that priming stimuli such as LPS also affect PTMs of NLRP3-associated proteins, thus adding an extra level of control of the inflammasome pathway downstream of NLRP3. 


\section{Protein Binding Partner Regulated Priming}

In addition to transcriptional and post-translational regulation mechanisms, NLRP3 priming and activation is both positively and negatively influenced by several protein binding partners.

\section{NEK7}

A genome-wide CRISPR screen conducted by Schmid-Burgk et al. (2015) identified NIMA-related kinase 7 (NEK7), a serine-threonine kinase, as a critical component in NLRP3 inflammasome activation ${ }^{77}$. Prior to this, NEK7 was known to play an important role in regulating mitosis and progression of the cell cycle ${ }^{78}$. NEK7 has since been described as a 'cellular switch' between mitosis and NLRP3 inflammasome activation as the quantity of NEK7 present in macrophages is insufficient to allow both to occur simultaneously ${ }^{78,79}$. NLRP3 priming with LPS and subsequent activation stimuli trigger an increase in NLRP3-NEK7 interaction, which is partly mediated through electrostatic complementarity as NLRP3 has an overall negative charge, while NEK7 is positively charged overall ${ }^{79}$. This interaction with NLRP3 is specific as NEK7 does not interact with NLRC4 or AIM2 ${ }^{78}$. Together, NLRP3 and NEK7 form a large oligomeric complex (>1,000 kDa) which leads to inflammasome assembly as NEK7 mediates the formation of bipartite interactions between adjacent NLRP3 subunits ${ }^{79,80}$. NEK7 is therefore essential for ASC speck formation, caspase- 1 activation, IL-1 $\beta$ release and pyroptosis in response to NLRP3 activation, as demonstrated in multiple in vitro and in vivo models ${ }^{77,78,80}$. He et al., (2016) identified potassium efflux induced by NLRP3 activators as an essential trigger to induce NEK7 to interact with NLRP3 ${ }^{80}$ while other studies have found that this interaction requires reactive oxygen species $(\mathrm{ROS})^{78}$, supporting a broad role for 
NEK7 in response to multiple NLRP3 inflammasome activators. Interestingly, NEK7 is also regulated by PTMs (Figure 3), with glutathione transferase omega-1 (GSTO11) mediating deglutathionylation of NEK7 to promote NLRP3 activation ${ }^{81}$. NEK7 is also phosphorylated by polo-like kinase 4 (PLK4) at Ser204 which attenuates NEK7NLRP3 interaction, therefore suppressing inflammasome activation ${ }^{82}$.

These studies suggest NEK7 is involved primarily in NLRP3 activation but a recent preprint study by Schmacke et al. (2019) argues that NEK7 functions as an NLRP3 priming factor ${ }^{83}$. Importantly, this study also identified a NEK7-independent mechanism of NLRP3 activation, involving TAK1-driven post-translational priming of NLRP3. The authors suggest that previous studies were conducted in murine models while their research utilised human induced pluripotent stem cell-derived macrophages as the explanation for these differing results. This study highlights the complexity of NLRP3 regulation via NEK7 and the need for further investigation, particularly as determining the precise role played by NEK7 could identify it as a therapeutic target in NLRP3 inflammasome-mediated diseases ${ }^{83-86}$.

\section{Pyrin-only proteins}

PYD-PYD interactions between NLRP3 and ASC are essential for inflammasome assembly but our understanding of how these are regulated is limited ${ }^{87,88}$. In the last two decades, a new family of proteins consisting of a single PYD have been discovered. These PYD-only proteins (POPs) are small cytoplasmic decoy proteins that regulate inflammasome activation in human cells, but they are not expressed in rodents (Figure 4) ${ }^{89-91}$.

POP1 has $88 \%$ similarity with the ASC PYD and can bind to the ASC PYD, preventing ASC self-polymerisation and interaction with the PYDs of pattern 
recognition receptors (PRRs) such as NLRP3 ${ }^{90}$. As a result, POP1 inhibits the formation of all ASC-dependent inflammasomes and subsequent caspase-1 activation and $\mathrm{IL}-1 \beta$ release $\mathrm{e}^{90,92}$. In addition, POP1 regulates the expression of NF$\mathrm{KB}$-inducible genes through suppressing the kinase activity of the IKK complex ${ }^{90}$. The expression of POP1 is increased in response to TLR4, TLR2, and IL-1 receptor signalling (4 hours post stimulation $)^{92}$. This indicates that POP1 functions as part of a negative feedback mechanism that allows for early inflammation and host defence but where long-term priming promotes the resolution of inflammasome-driven inflammation. Transgenic mouse studies have demonstrated that POP1 protects against systemic inflammation and can regulate models of NLRP3-driven diseases including one caused by a CAPS-associated mutation ${ }^{92}$. Interestingly, individuals with CAPS exhibit reduced POP1 expression which could contribute to the uncontrolled NLRP3 inflammasome-driven inflammation they experience ${ }^{92}$. Despite having low similarity with the ASC PYD (37\%), POP2 also interacts with ASC and prevents activation of ASC-dependent inflammasomes ${ }^{89,93,94}$. In contrast to POP1, POP2 also interacts directly with PYD-containing NLRs, including NLRP3, and therefore may provide more versatile inflammasome regulation ${ }^{94}$. POP2 also regulates NLRP3 inflammasome transcriptional priming and production of NF-kBdependent cytokines, including TNF- $\alpha$ and IL-6. This occurs as POP2 reduces TLRinduced NF-kB signalling at the level of p65 (RelA), resulting in less nuclear import and altered accumulation of NF-kB ${ }^{89,95}$. Similar to POP1, POP2 is a late-response inflammatory regulator induced by pro-inflammatory stimuli (peaking at $20 \mathrm{~h}$ postLPS stimulation) thus participating in resolution of inflammation, and again suggesting long-term priming is anti-inflammatory ${ }^{95}$. Anti-inflammatory stimuli, such 
as IL-10, also increase POP2 expression, further implicating POP2 in the maintenance of an anti-inflammatory milieu ${ }^{95}$.

POP3 shares only $18.9 \%$ sequence identity with the PYD of ASC ${ }^{91}$. Khare et al. (2014) demonstrated that POP3 does not regulate NF-KB or the NLRP3 inflammasome but instead competes with ASC to bind directly to the PYD of two other inflammasome sensor proteins, AIM2 and Interferon-y Inducible Protein 16 (IFI16). POP3 therefore functions as a specific inhibitor of AIM2-like receptors in response to DNA virus infection ${ }^{91}$.

POP4 was initially characterised as a pseudogene, but the POP4 gene has been shown to code for a functional protein expressed widely in human cells, with increased expression in monocytic cells upon exposure to inflammatory stimuli, such as LPS ${ }^{96}$. POP4 inhibits NF-KB by reducing the phosphorylation of p65 which limits its transcriptional activity and prevents production of NF-kB-dependent cytokines. However, POP4 does not appear to inhibit inflammasome activation as it does not affect NLRP3 or ASC-mediated release of IL-1 $\beta^{96}$. POP4 is therefore considered to have a more exclusive role in regulating NF-kB compared to the other $\mathrm{POPs}^{96}$. Although POP4 could subsequentially affect NLRP3 transcriptional priming, this has not been demonstrated.

POPs are therefore involved in NLRP3 priming via direct interactions with NLRP3 and ASC, and indirectly via effects on NF-KB which may affect both transcriptional priming and also potentially NLRP3 PTMs.

\section{CARD-only proteins (COPs)}

Three CARD-only proteins (COPs) have been identified in humans; CARD16, CARD17 and CARD18, similar to POPs they are not expressed in rodents ${ }^{97-99}$. 
Although CARD interactions are essential for NLRP3 inflammasome assembly, the regulatory roles played by these different COPs currently remain somewhat unclear $(\text { Figure } 4)^{87,88}$.

CARD16 (Pseudo-ICE or Cop) has 97\% nucleic acid sequence identity to the procaspase-1 CARD, allowing strong and specific interactions, without binding to other CARD-containing proteins ${ }^{98,99}$. Binding of CARD16 to pro-caspase-1 prevents CARD-CARD interactions, thereby preventing functional inflammasome formation ${ }^{99}$. CARD16 may also play a role in activating NF-KB, potentially through receptorinteracting-serine/threonine-protein kinase 2 (RIP2) which has been shown to interact with the IKK complex and could therefore affect NLRP3 priming in certain contexts, although this has not been characterised ${ }^{99,100}$. CARD16 is expressed widely in human cells and its expression is comparable to that of caspase-1, suggesting they may be under similar transcriptional regulation at basal levels ${ }^{99,100}$. Although these studies suggest CARD16 is anti-inflammatory, a report from Karasawa et al. (2015) argues that the oligomerisation of CARD16 promotes caspase- 1 activation and IL-1 $1 \beta$ release ${ }^{101}$. Therefore, additional investigations are necessary to elucidate the role of CARD16 in NLRP3 inflammasome regulation. CARD17 (Inca; Inhibitory CARD) has $81 \%$ sequence identity with the CARD of procaspase- 1 and has been found to bind to this CARD, preventing IL-1 $\beta$ release in response to inflammatory stimuli ${ }^{98}$. A study from Lu et al. (2016) found CARD17 does not bind to monomeric pro-caspase-1 but instead caps the growing oligomeric form to prevent caspase-1 polymerisation and inflammasome formation ${ }^{102}$. CARD16 and CARD17 may thus have different roles in regulating caspase-1 activation ${ }^{101,102}$. CARD17 is co-expressed with pro-caspase- 1 in a range of human cells, and like procaspase- 1 its expression is upregulated in monocytes in response to IFN- $\gamma^{98}$. 
CARD18 (ICEBERG) shares only 53\% sequence identity with the CARD of procaspase-1, but can specifically interact with caspase- 1 and reduce $\mathrm{IL}-1 \beta$ release in response to NLRP3 inflammatory stimuli99. CARD18 acts as a competitive antagonist and preferentially binds to caspase-1, inhibiting RIP2-mediated caspase-1 oligomerisation ${ }^{97,99,102}$. However, unlike CARD16, CARD18 cannot bind directly to RIP2 and is therefore a less potent inhibitor ${ }^{97,99}$. Expression of CARD18 is increased after 4-6 hours stimulation with LPS or TNF in THP-1 cells and monocytes, therefore after initial inflammasome activation, CARD18 is induced as a negative feedback mechanism to regulate IL-1 $\beta$ production ${ }^{97}$. However, a contradictory report observed that CARD18 oligomerises and promotes formation of caspase-1 filaments and is therefore incapable of inhibiting inflammasome formation ${ }^{102}$. This may be because CARD18 levels induced by doxycycline treatment were insufficient in these experiments or perhaps binding of CARD18 to caspase- 1 may be regulated by other cellular proteins. Further study is thus required to define the role of CARD18. POPs and COPs are clearly important regulators of the NLRP3 inflammasome that are influenced by NLRP3 priming stimuli. However, our knowledge of the physiological function of these proteins remains somewhat limited. This is likely because they are only expressed in the primate lineage and are not expressed in mice, suggesting inflammasome regulation is more complex in humans than in rodents $^{92,101}$. Systemic inflammatory responses such as fever are essential for host defence but come at a huge metabolic cost, while sustained inflammation also causes tissue damage ${ }^{103}$. POPs and COPs are an additional checkpoint in primates that can restrain excessive inflammation, thus preventing damage to the host and aiding a return to homeostasis ${ }^{87}$. The absence of this checkpoint in mice suggests that rodents may have evolved other mechanisms to restrain damaging 
inflammation. This may include the expression of alternative splice variants of proteins that can limit inflammatory signalling ${ }^{104}$. For example, alternative splice forms of ASC have been shown to inhibit inflammasome activation ${ }^{105}$. Transgenic mouse models have offered opportunities for studying POPs in vivo and have discovered novel regulatory functions ${ }^{91-93}$. However, there are currently no COP transgenic mouse models and there remains the need for the development of human models to study inflammasome regulation via both COPs and POPs.

\section{Therapeutic Potential of Targeting NLRP3 Priming and PTMs}

Due to its association with the pathogenesis of a wide range of human diseases, NLRP3 is an attractive target for the development of new anti-inflammatory therapies ${ }^{6,106,107}$. Small molecule inhibitors of NLRP3 such as MCC950 (CP-456,773) have been extensively studied in a huge number of disease models with very promising results ${ }^{6,108}$. Indeed, NLRP3 inhibitors have recently entered clinical trials for safety and efficacy marking the advent of an exciting new era for inflammasome research, where the potential of targeting this pathway for the treatment of inflammatory diseases may be realised ${ }^{107,109}$. MCC950 is a non-covalent inhibitor that directly interacts with NLRP3 and blocks its ATPase activity thereby preventing inflammasome formation in response to all NLRP3 priming and activation stimuli ${ }^{110,111}$. Directly inhibiting NLRP3 is advantageous in terms of specificity, but as we have highlighted in previous sections, there are many proteins involved in NLRP3 priming that could be targeted to prevent NLRP3-driven inflammation.

\section{Targeting transcriptional priming of the NLRP3 inflammasome}


Transcriptional priming of the NLRP3 inflammasome will be affected by compounds

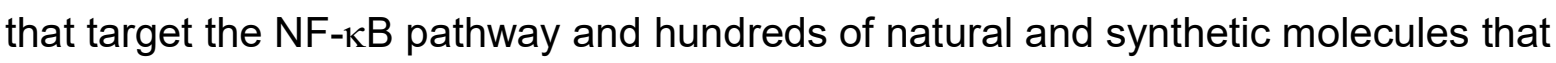

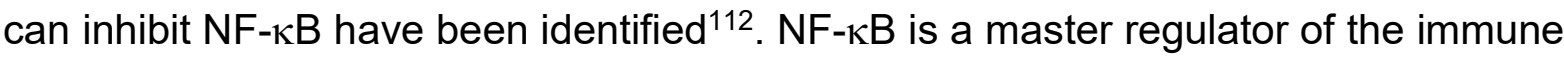
response and is induced by many different stimuli ${ }^{113}$, thus parsing the relative impact of these inhibitors on NLRP3 signalling is challenging as they will broadly impact inflammation. However, some NF-kB inhibitors have also been shown to inhibit NLRP3 more directly. The vinyl sulfone BAY 11-7082 is an IKK $\beta$ inhibitor but was also shown to block NLRP3 activation distinct from any effect on transcriptional priming, likely through inhibiting the ATPase activity of NLRP3 ${ }^{114,115}$. BAY 11-7082 is however a non-specific inhibitor as it has also been observed to potently block protein tyrosine phosphatases ${ }^{116}$.

It can be argued that the production of mature IL-1 $\beta$ is the most important NLRP3dependent signal. Individuals with CAPS, where inflammation is driven by mutations in NLRP3, are currently treated with anti-IL-1 biologics which are very effective ${ }^{117}$. This effectiveness may be due to the positive feed-back loop of IL-1 $\beta$ in vivo, where IL-1 $\beta$ can induce its own gene transcription ${ }^{118,119}$ and IL-1 $\beta$ can also prime NLRP3 for activation ${ }^{120}$. The transcription of pro-IL-1 $\beta$ is highly regulated, including by metabolic adaptations. LPS signaling in macrophages drives a shift to aerobic glycolysis that appears to be critical for pro-IL-1 $\beta$ synthesis, as inhibiting glycolysis with 2-deoxyglucose suppresses IL-1 $\beta$ mRNA ${ }^{121,122}$. Further studies have identified multiple metabolites and metabolic pathways that regulate LPS induced pro-IL-1 $\beta$ production $^{123}$. Targeting these metabolic pathways may indirectly impact NLRP3 transcriptional priming.

\section{Targeting NLRP3 phosphorylation}


The identification of priming associated PTMs of NLRP3 has provided a new strategy for modulating NLRP3 activity. Kinases have been intensively studied as drug targets for decades, and the United States Food and Drug Administration has approved $>60$ kinase inhibitors to date, of which the vast majority are cancer treatments. NLRP3 activation requires serine phosphorylations mediated by JNK1 and PKD, therefore inhibition of these kinases should limit NLRP3 activation. The ATP competitive JNK inhibitor SP600125 was shown to block NLRP3 activation ${ }^{30}$ and second generation JNK inhibitors have been evaluated in the clinic ${ }^{124}$, including the Celgene molecule CC-90001 for the treatment of idiopathic pulmonary fibrosis ${ }^{125}$. A number of PKD inhibitors (e.g. (CRT 0066101 and kb NB 142-70) were found to inhibit NLRP3 activation ${ }^{47}$ although there are no PKD inhibitors that have advanced to clinical trials. NLRP3 is also phosphorylated at Ser298 by PKA and this phosphorylation inhibits NLRP3 activity ${ }^{48}$. PKA is activated by cAMP so increasing cAMP levels using synthetic cAMP molecules such as dibutyryl cAMP can indirectly prevent NLRP3 activation. Other studies have shown that modulating cAMP with adenylate cyclase activators or phosphodiesterase inhibitors can inhibit NLRP3 although this effect was not attributed to $\mathrm{PKA}^{126,127}$.

Several additional NLRP3 phosphorylation sites at Ser5, Tyr32 and Tyr861 appear to inhibit NLRP3 activity 49,51,53. The kinases responsible for these modifications are currently unknown and so the potential impact of modulating their activity remains to be determined. However, the phosphatases involved in dephosphorylation of these sites have been identified as PP2A, PTEN and PTPN22 ${ }^{49,51,53}$, and inhibiting these phosphatases will likely limit NLRP3 activation. Phosphatases were previously considered to be difficult drug targets, however a number of phosphatase inhibitors are now being evaluated in clinical trials ${ }^{128}$. The PP2A inhibitor okadaic acid can 
inhibit NLRP3 activation ${ }^{49}$ and the PP2A inhibitor LB-100 is currently in clinical development ${ }^{129}$. In one of these trials (NCT03886662) LB-100 is being evaluated for the treatment of myelodysplastic syndrome (MDS). Interestingly, a previous study by Basiorka et al. (2016) found that NLRP3 drives pyroptosis and ineffective haematopoiesis in MDS ${ }^{130}$. LB-100 may thus have both anti-cancer and anti-NLRP3 effects in MDS.

\section{Targeting NLRP3 ubiquitination}

The numerous ubiquitin modifications associated with NLRP3 could also be targeted to modulate NLRP3 activity. The DUBs BRCC3, USP7 and USP47 appear to be essential for NLRP3 activation ${ }^{36,39}$. Consistent with this, various inhibitors of DUBs such as G5, PR619, bAP15 and WP1130 can all block NLRP3 activation²6,35,36. Specific DUB inhibitors have made promising pre-clinical progress for disease indications in oncology, immuno-oncology, neurodegeneration and inflammation ${ }^{131}$. Of these USP7, inhibitors may be the most promising they have been shown to inhibit NLRP3 ${ }^{39}$, a number are in preclinical development ${ }^{131}$, and highly specific inhibitors are available ${ }^{39,132}$. Multiple E3 ligases are also linked to NLRP3 function, as described above they can mediate NLRP3 degradation and inactivation but can also positively regulate NLRP3. E3 ligases are a very large family of proteins and although E3 ligase inhibitors are in use clinically, for example Birinapant which blocks the inhibitors of apoptosis proteins (IAPs), no inhibitors of the E3 ligases associated with NLRP3 have been developed ${ }^{132,133}$.

\section{Targeting additional NLRP3 PTMs}


Additional PTMs of NLRP3 such as sumoylation may also be interesting targets. Desumoylation of NLRP3 by SENP6 and SENP7 could be inhibited to decrease NLRP3 activity ${ }^{54}$. SENP inhibitors have been described, although most target SENP1 and SENP2, a couple with activity towards SENP6 and SENP7 were identified but these have not been well characterised ${ }^{134}$. Alternatively, it has been proposed that sumoylation activates NLRP3 ${ }^{55}$, in this case SUMO inhibitors will block NLRP3. The SUMO inhibitor TAK-981 (ML-792) was developed by Takeda ${ }^{135}$ and is currently being evaluated in a clinical trial (NCT04074330) for non-Hodgkin lymphoma ${ }^{136}$.

Recently it was demonstrated that acetylation is required for NLRP3 inflammasome assembly ${ }^{59,60}$. These studies suggest that this acetylation is mediated by the acetyl transferase KAT5 (Tip60) ${ }^{60}$ and deacetylation is mediated by SIRT2 ${ }^{59}$. Thus, a SIRT2 activator could negatively regulate NLRP3, although SIRT1 activators have been developed none have been characterised for SIRT2 ${ }^{137}$. The KAT5 inhibitor NU9056 was found to decrease NLRP3 activation in vitro and in vivo ${ }^{60}$. However, few histone acetyl transferase inhibitors have been developed and many of those that have are thiol-reactive and have off-target effects ${ }^{138}$. Alkylation of NLRP3 by BOT-4-one attenuates its activation by interfering with ATPase activity and enhancing ubiquitination ${ }^{61}$. However, similar to Bay 11-7082, BOT-4-one is also an NF- $\mathrm{KB}$ inhibitor ${ }^{139}$ and also partially blocks NLRC4 ${ }^{61}$.

\section{Targeting protein-protein interactions}

An additional approach to target NLRP3 priming could be to disrupt protein-protein interactions (PPI) with NLRP3 protein binding partners. Recognising the importance of PPIs to the function of biological systems, medicinal chemists have made 
considerable progress in their ability to disrupt these interactions with small molecules ${ }^{133,140}$. The development of PPI inhibitors and in particular intracellular ones is challenging. There are however a number of successful examples such as ABT-199 (Venetoclax) which targets B-cell lymphoma 2 (Bcl-2) protein interactions and is used to treat chronic lymphocytic leukemia ${ }^{133,140}$. Molecules that could disrupt NLRP3 oligomerisation, NLRP3-NEK7 or NLRP3-ASC interactions would effectively limit NLRP3 activation. There is unfortunately a relatively limited amount of structural data available for NLRP3 that could inform the design of such molecules. The NLRP3 PYD domain crystal structure was published in $2011^{141}$ and a cryo-EM structure of NLRP3 (without the PYD) and NEK7 was published in 201979. The study by Sharif et al. (2019) ${ }^{79}$ reveals potential NLRP3-NEK7 interaction interfaces that could be disrupted by PPI inhibitors.

An alternative to small molecule inhibitors could be to administer or enhance the expression of endogenous regulators such as POP1 or POP2 that can disrupt NLRP3-ASC interactions ${ }^{87,142}$. Intriguingly, a monoclonal antibody to human ASC known as IC100 has been reported to be taken up by neurons and THP-1 cells ${ }^{143}$ suggesting that antibodies could potentially be used to disrupt NLRP3 inflammasome formation.

The discoveries of NLRP3 PTMs and protein binding partners have provided new potentially druggable targets for NLRP3-driven diseases (summarized in Figure 5). Some of these targets, particularly in the kinase space, may already have well characterised inhibitors that could allow the repurposing of molecules which have already been successful in clinical development.

\section{Concluding Remarks and Open Questions}


NLRP3 priming can now be clearly divided into transcriptional priming that is not essential for NLRP3 activation, and post-translational modification-mediated priming that is essential for the formation of the NLRP3 inflammasome. NLRP3 priming can be further influenced by inflammasome protein binding partners including NEK7, POPs and COPs. There are however still numerous outstanding questions related to NLRP3 priming.

It is unclear how all of the NLRP3 PTMs, such as multiple phosphorylation and ubiquitination events, are spatially and temporally co-ordinated. As the mechanism of NLRP3 inflammasome assembly is not understood ${ }^{3,4}$, it is uncertain whether these PTMs may affect specific steps in this process, such as NLRP3 oligomerisation or structural changes that may be required for NLRP3 activation ${ }^{34}$. Future studies on the structure of the NLRP3 inflammasome will likely clarify some of these events. Another enigmatic aspect of NLRP3 priming is that human monocytes appear to break all the rules that were established by studies in macrophages. It has long been appreciated that human monocytes can activate NLRP3 in response to LPS alone ${ }^{144}$. This pathway appears to require relatively long stimulation with LPS and has been termed alternative NLRP3 activation ${ }^{9}$. A recent report by Gritsenko et al. $(2020)^{145}$ has observed that in human monocytes caspase-1 activation and substrate processing (IL-18, GSDMD) are detected in response to nigericin stimulation in the absence of any classical priming stimulus. These results suggest priming is not essential for NLRP3 activation in human monocytes. Thus, the two-step activation dogma does not apply to monocytic cells, highlighting the disparities in NLRP3 activation mechanisms in different cell types.

A recent study by Hoss et al. (2019) ${ }^{146}$ discovered that the human NLRP3 gene is regulated by alternative splicing, resulting in the expression of an isoform lacking 
exon 5. The loss of this exon results in a shorter LRR domain which disrupts NLRP3NEK7 interaction and prevents NLRP3 inflammasome activation. The relative expression of these NLRP3 isoforms thus represents another layer of regulation in the NLRP3 signalling pathway ${ }^{146}$. How priming stimuli may influence the balance of the active full-length or inactive $\Delta$ exon 5 isoforms is currently not understood. It is clear that the human NLRP3 inflammasome is subject to additional regulatory mechanisms that are absent in mice. In particular, POPs, COPs and alternative splicing may significantly influence NLRP3 activation in human cells. In addition, there is an appreciable divergence between innate immune signalling pathways in human and mouse cells ${ }^{147,148}$ that may affect how stimuli such as LPS trigger NLRP3 priming.

Despite significant progress in the field the molecular mechanisms of NLRP3 priming remain somewhat mysterious. A deeper understanding of this process will inform our understanding of the physiological role of NLRP3 ${ }^{149}$ and will be essential to the development of novel treatments for NLRP3-driven diseases. 


\section{Authorship}

C.M.M. and R.C.C. conceptualised this review, wrote and edited the text, and created the figures. All authors agreed to the submission of the article.

\section{Acknowledgements}

C.M.M. is funded by a Department of the Economy Northern Ireland PhD

Studentship. R.C.C. is supported by institutional funding from the Wellcome-Wolfson Institute for Experimental Medicine and Queen's University Belfast. Figures were created with BioRender.com.

\section{Conflict of Interest Disclosure}

R.C.C. is a co-inventor on a patent (US 10,538,487; EP 3,259,253) and patent applications (WO2018215818, WO2017140778) for NLRP3 inhibitors, which are licensed to Inflazome Ltd, a company headquartered in Dublin, Ireland. Inflazome is developing drugs that target the NLRP3 inflammasome to address unmet clinical needs in inflammatory disease.

\section{References}

1. Schroder K, Tschopp J. The Inflammasomes. Cell. 2010;140:821-832.

2. Hoffman HM, Mueller JL, Broide DH, et al. Mutation of a new gene encoding a putative pyrin-like protein causes familial cold autoinflammatory syndrome and 
Muckle-Wells syndrome. Nat Genet. 2001;29:301-305.

3. Próchnicki T, Mangan MS, Latz E. Recent insights into the molecular mechanisms of the NLRP3 inflammasome activation. F1000Res. 2016;5:F1000 Faculty Rev-1469.

4. Swanson K V., Deng M, Ting JPY. The NLRP3 inflammasome: molecular activation and regulation to therapeutics. Nat Rev Immunol. 2019;19(8):477489.

5. Boucher D, Monteleone M, Coll RC, et al. Caspase-1 self-cleavage is an intrinsic mechanism to terminate inflammasome activity. $J$ Exp Med. 2018;215:827-840.

6. Mangan MSJ, Olhava EJ, Roush WR, et al. Targeting the NLRP3 inflammasome in inflammatory diseases. Nat Rev Drug Discov. 2018;17:588606.

7. Muñoz-Planillo R, Kuffa P, Martínez-Colón $G$, et al. $K+$ Efflux Is the Common Trigger of NLRP3 Inflammasome Activation by Bacterial Toxins and Particulate Matter. Immunity. 2013;38:1142-1153.

8. Groß CJ, Mishra R, Schneider KS, et al. K+ Efflux-Independent NLRP3 Inflammasome Activation by Small Molecules Targeting Mitochondria. Immunity. 2016;45(4):761-773

9. Gaidt MM, Ebert TS, Chauhan D, et al. Human Monocytes Engage an Alternative Inflammasome Pathway. Immunity. 2016;44:833-846.

10. Chen J, Chen ZJ. Ptdlns4P on dispersed trans-Golgi network mediates NLRP3 inflammasome activation. Nature. 2018;564:71-76.

11. Kelley N, Jeltema D, Duan Y, et al. The NLRP3 inflammasome: An overview of mechanisms of activation and regulation. Int J Mol Sci. 2019;20(13):3328.

12. Patel MN, Carroll RG, Galván-Peña S, et al. Inflammasome Priming in Sterile Inflammatory Disease. Trends Mol Med. 2017;23:165-180.

13. Prime / Definition of Prime by Merriam-Webster Available from: https://www.merriam-webster.com/dictionary/prime\#h3. Accessed May 15, 2020.

14. Martinon F, Burns K, Rg Tschopp J. The Inflammasome: A Molecular Platform Triggering Activation of Inflammatory Caspases and Processing of prolL-beta. Mol Cell. 2002;10:417-426.

15. Agostini L, Martinon F, Burns K, et al. NALP3 forms an IL-1ß-processing inflammasome with increased activity in Muckle-Wells autoinflammatory disorder. Immunity. 2004;20:319-325.

16. Garlanda C, Dinarello CA, Mantovani A. The Interleukin-1 Family: Back to the Future. Immunity. 2013;39:1003-1018.

17. Perregaux D, Barberia J, Lanzetti AJ, et al. IL-1 beta maturation: evidence that mature cytokine formation can be induced specifically by nigericin. $\mathrm{J}$ Immunol. 1992;149:1294-1303.

18. Hogquist KA, Nett MA, Unanue ER, et al. Interleukin 1 is processed and released during apoptosis. Proc Natl Acad Sci U S A. 1991;88:8485-8489.

19. Perregaux D, Gabel CA. Interleukin-1 $\beta$ maturation and release in response to ATP and nigericin. Evidence that potassium depletion mediated by these agents is a necessary and common feature of their activity. $\mathrm{J}$ Biol Chem. 1994;269:15195-15203.

20. Mariathasan S, Weiss DS, Newton K, et al. Cryopyrin activates the inflammasome in response to toxins and ATP. Nature. 2006;440:228-232.

21. Kuida K, Lippke JA, Ku G, et al. Altered cytokine export and apoptosis in mice 
deficient in interleukin-1 $\beta$ converting enzyme. Science. 1995;267:2000-2003.

22. Zhu Q, Kanneganti T-D. Cutting Edge: Distinct Regulatory Mechanisms Control Proinflammatory Cytokines IL-18 and IL-1 $\beta$. J Immunol. 2017; 198:4210-4215.

23. O'Connor W, Harton JA, Zhu X, et al. Cutting Edge:

CIAS1/Cryopyrin/PYPAF1/NALP3/ CATERPILLER 1.1 Is an Inducible Inflammatory Mediator with NF-kB Suppressive Properties. J Immunol. 2003;171:6329-6333.

24. Bauernfeind FG, Horvath G, Stutz A, et al. Cutting Edge: NF-kB Activating Pattern Recognition and Cytokine Receptors License NLRP3 Inflammasome Activation by Regulating NLRP3 Expression. J Immunol. 2009;183:787-791.

25. Bezbradica JS, Coll RC, Schroder K. Sterile signals generate weaker and delayed macrophage NLRP3 inflammasome responses relative to microbial signals. Cell Mol Immunol. 2017;14:118-126.

26. Juliana C, Fernandes-Alnemri T, Kang S, et al. Non-transcriptional priming and deubiquitination regulate NLRP3 inflammasome activation. J Biol Chem. 2012;287:36617-36622.

27. Schroder K, Sagulenko V, Zamoshnikova A, et al. Acute lipopolysaccharide priming boosts inflammasome activation independently of inflammasome sensor induction. Immunobiology. 2012;217:1325-1329.

28. Fernandes-Alnemri T, Kang S, Anderson C, et al. Cutting Edge: TLR Signaling Licenses IRAK1 for Rapid Activation of the NLRP3 Inflammasome. J Immunol. 2013;191:3995-3999.

29. Lin KM, Hu W, Troutman TD, et al. IRAK-1 bypasses priming and directly links TLRs to rapid NLRP3 inflammasome activation. Proc Natl Acad Sci U S A. 2014;111:775-780.

30. Song N, Liu ZS, Xue W, et al. NLRP3 Phosphorylation Is an Essential Priming Event for Inflammasome Activation. Mol Cell. 2017;68(1):185-197.e6.

31. O'Neill LAJ, Bowie AG. The family of five: TIR-domain-containing adaptors in Toll-like receptor signalling. Nat Rev Immunol. 2007;7:353-364.

32. Gong YN, Wang X, Wang J, et al. Chemical probing reveals insights into the signaling mechanism of inflammasome activation. Cell Res. 2010;20:1289_ 1305.

33. Baker PJ, De Nardo D, Moghaddas F, et al. Posttranslational modification as a critical determinant of cytoplasmic innate immune recognition. Physiol Rev. 2017;97:1165-1209.

34. Sandall CF, MacDonald JA. Effects of phosphorylation on the NLRP3 inflammasome. Arch. Biochem. Biophys. 2019;670:43-57.

35. Lopez-Castejon G, Luheshi NM, Compan V, et al. Deubiquitinases regulate the activity of caspase- 1 and interleukin- $1 \beta$ secretion via assembly of the inflammasome. J Biol Chem. 2013;288:2721-2733.

36. Py BF, Kim MS, Vakifahmetoglu-Norberg $\mathrm{H}$, et al. Deubiquitination of NLRP3 by BRCC3 Critically Regulates Inflammasome Activity. Mol Cell. 2013;49:331338.

37. Ren $\mathrm{G}$, Zhang X, Xiao $\mathrm{Y}$, et al. ABRO1 promotes NLRP3 inflammasome activation through regulation of NLRP3 deubiquitination. EMBO J; 2019;38(6):e100376.

38. Wang W, Hu D, Wu C, et al. STING promotes NLRP3 localization in ER and facilitates NLRP3 deubiquitination to activate the inflammasome upon HSV-1 infection. PLOS Pathog. 2020;16:e1008335. 
39. Palazón-Riquelme P, Worboys JD, Green J, et al. USP7 and USP47 deubiquitinases regulate NLRP3 inflammasome activation. EMBO Rep. 2018;19(10):e44766

40. Han SH, Lear TB, Jerome JA, et al. Lipopolysaccharide primes the NALP3 inflammasome by inhibiting its ubiquitination and degradation mediated by the SCFFBXL2 E3 ligase. J Biol Chem. 2015;290:18124-18133.

41. Song $\mathrm{H}$, Liu B, Huai $\mathrm{W}$, et al. The E3 ubiquitin ligase TRIM31 attenuates NLRP3 inflammasome activation by promoting proteasomal degradation of NLRP3. Nat Commun. 2016;7:13727.

42. Kawashima A, Karasawa T, Tago K, et al. ARIH2 Ubiquitinates NLRP3 and Negatively Regulates NLRP3 Inflammasome Activation in Macrophages. $J$ Immunol. 2017;199:3614-3622.

43. Tang J, Tu S, Lin G, et al. Sequential ubiquitination of NLRP3 by RNF125 and Cbl-b limits inflammasome activation and endotoxemia. J Exp Med. 2020;217(4):e20182091.

44. Yan Y, Jiang W, Liu L, et al. Dopamine controls systemic inflammation through inhibition of NLRP3 inflammasome. Cell. 2015;160:62-73.

45. Wan $\mathrm{P}$, Zhang $\mathrm{Q}$, Liu W, et al. Cullin1 binds and promotes NLRP3 ubiquitination to repress systematic inflammasome activation. FASEB J. 2019;33:5793-5807.

46. Humphries $F$, Bergin $R$, Jackson $R$, et al. The E3 ubiquitin ligase Pellino2 mediates priming of the NLRP3 inflammasome. Nat Commun. 2018;9:1560.

47. Zhang Z, Meszaros G, He WT, et al. Protein kinase D at the Golgi controls NLRP3 inflammasome activation. J Exp Med. 2017;214:2671-2693.

48. Mortimer L, Moreau F, MacDonald JA, et al. NLRP3 inflammasome inhibition is disrupted in a group of auto-inflammatory disease CAPS mutations. Nat Immunol. 2016;17:1176-1186.

49. Stutz A, Kolbe CC, Stahl R, et al. NLRP3 inflammasome assembly is regulated by phosphorylation of the pyrin domain. J Exp Med. 2017;214:1725-1736.

50. Mao L, Kitani A, Hiejima E, et al. Bruton tyrosine kinase deficiency augments NLRP3 inflammasome activation and causes IL-1 $\beta$-mediated colitis. J Clin Invest. 2020;130(4):1793-1807

51. Spalinger MR, Kasper S, Gottier C, et al. NLRP3 tyrosine phosphorylation is controlled by protein tyrosine phosphatase PTPN22. J Clin Invest. 2016;126:1783-1800.

52. Spalinger MR, Lang S, Gottier C, et al. PTPN22 regulates NLRP3-mediated IL1B secretion in an autophagy-dependent manner. Autophagy. 2017;13:1590-1601.

53. Huang $\mathrm{Y}$, Wang $\mathrm{H}$, Hao $\mathrm{Y}$, et al. Myeloid PTEN promotes chemotherapyinduced NLRP3-inflammasome activation and antitumour immunity. Nat Cell Biol. 2020;1-12.

54. Barry R, John SW, Liccardi G, et al. SUMO-mediated regulation of NLRP3 modulates inflammasome activity. Nat Commun. 2018;9(1):3001

55. Shao L, Liu Y, Wang W, et al. SUMO1 SUMOylates and SENP3 deSUMOylates NLRP3 to orchestrate the inflammasome activation. FASEB J. 2020;34:1497-1515.

56. Mishra BB, Rathinam VAK, Martens GW, et al. Nitric oxide controls the immunopathology of tuberculosis by inhibiting NLRP3 inflammasomedependent processing of IL-1 $\beta$. Nat Immunol. 2013;14:52-60.

57. Mao K, Chen S, Chen M, et al. Nitric oxide suppresses NLRP3 inflammasome 
activation and protects against LPS-induced septic shock. Cell Res. 2013;23:201-212.

58. Hernandez-Cuellar E, Tsuchiya K, Hara H, et al. Cutting Edge: Nitric Oxide Inhibits the NLRP3 Inflammasome. J Immunol. 2012;189:5113-5117.

59. He M, Chiang HH, Luo H, et al. An Acetylation Switch of the NLRP3 Inflammasome Regulates Aging-Associated Chronic Inflammation and Insulin Resistance. Cell Metab. 2020;31:580-591.e5.

60. Zhao K, Zhang Y, Xu X, et al. Acetylation is required for NLRP3 selfaggregation and full activation of the inflammasome. bioRxiv 2019.12.31.891556; doi: https://doi.org/10.1101/2019.12.31.891556

61. Shim DW, Shin WY, Yu SH, et al. BOT-4-one attenuates NLRP3 inflammasome activation: NLRP3 alkylation leading to the regulation of its ATPase activity and ubiquitination. Sci Rep. 2017;7:1-12.

62. Bose S, Segovia JA, Somarajan SR, et al. ADP-Ribosylation of NLRP3 by Mycoplasma pneumoniae CARDS toxin regulates inflammasome activity. MBio. 2014;5(6):e02186-14.

63. Lee JY, Seo D, You J, et al. The deubiquitinating enzyme, ubiquitin-specific peptidase 50 , regulates inflammasome activation by targeting the ASC adaptor protein. FEBS Letters. 2017;591:479-490.

64. Xu Q, Xia W, Gu J, et al. Ubiquitination via the E3 Ligase TRAF3 by Targeting ASC for K63-Linked MAVS Promotes Inflammasome Activation. J Immunol. 2015;194(10):4880-90.

65. Rodgers MA, Bowman JW, Fujita $\mathrm{H}$, et al. The linear ubiquitin assembly complex (LUBAC) is essential for NLRP3 inflammasome activation. $J$ Exp Med. 2014;211:1333-1347.

66. Martin BN, Wang C, Willette-Brown J, et al. IKKa negatively regulates ASCdependent inflammasome activation. Nat Commun. 2014;5:1-14.

67. McAndrew CC, Napolitano NM, Vompe AD, et al. ASC phosphorylation at Y146 regulates inflammasome activation and pyroptosis. J Immunol;200.

68. Hara H, Tsuchiya K, Kawamura I, et al. Phosphorylation of the adaptor ASC acts as a molecular switch that controls the formation of speck-like aggregates and inflammasome activity. Nat Immunol. 2013;14:1247-1255.

69. Chung IC, OuYang CN, Yuan SN, et al. Pyk2 activates the NLRP3 inflammasome by directly phosphorylating ASC and contributes to inflammasome-dependent peritonitis. Sci Rep. 2016;6:36214.

70. Mambwe B, Neo K, Javanmard Khameneh H, et al. Tyrosine Dephosphorylation of ASC Modulates the Activation of the NLRP3 and AIM2 Inflammasomes. Front Immunol. 2019;10:1556.

71. Duong $\mathrm{BH}$, Onizawa M, Oses-Prieto JA, et al. A20 Restricts Ubiquitination of Pro-Interleukin-1 $\beta$ Protein Complexes and Suppresses NLRP3 Inflammasome Activity. Immunity. 2015;42:55-67.

72. Labbé K, McIntire CR, Doiron K, et al. Cellular Inhibitors of Apoptosis Proteins cIAP1 and clAP2 Are Required for Efficient Caspase-1 Activation by the Inflammasome. Immunity. 2011;35:897-907.

73. Xing Y, Yao X, Li H, et al. Cutting Edge: TRAF6 Mediates TLR/IL-1R Signaling-Induced Nontranscriptional Priming of the NLRP3 Inflammasome. $J$ Immunol. 2017;199:1561-1566.

74. Douglas T, Saleh M. Cross-regulation between LUBAC and caspase-1 modulates cell death and inflammation. J Biol Chem. 2020;295(16):5216-5228.

75. Hancz D, Westerlund E, Valfridsson C, et al. Streptolysin O Induces the 
Ubiquitination and Degradation of Pro-IL-1ß. J Innate Immun. 2019;11:457468.

76. Basak C, Pathak SK, Bhattacharyya A, et al. NF-kB- and C/EBP $\beta$-driven interleukin-1 $\beta$ gene expression and PAK1-mediated caspase-1 activation play essential roles in interleukin- $1 \beta$ release from Helicobacter pylori lipopolysaccharide-stimulated macrophages. J Biol Chem. 2005;280:4279_ 4288.

77. Schmid-Burgk JL, Chauhan D, Schmidt T, et al. A Genome-wide CRISPR Screen Identifies NEK7 as an Essential Component of NLRP3 Inflammasome Activation. J Biol Chem. 2016;291(1):103-9.

78. Shi $\mathrm{H}$, Wang $\mathrm{Y}$, Li X, et al. NLRP3 activation and mitosis are mutually exclusive events coordinated by NEK7, a new inflammasome component. Nat Immunol. 2016;17:250-258.

79. Sharif H, Wang li, li Wang W, et al. Structural mechanism for NEK7-licensed activation of NLRP3 inflammasome. Nature. 2019;570:338-343.

80. He Y, Zeng MY, Yang D, et al. NEK7 is an essential mediator of NLRP3 activation downstream of potassium efflux. Nature. 2016;530:354-357.

81. Hughes MM, Hooftman A, Angiari S, et al. Glutathione Transferase Omega-1 Regulates NLRP3 Inflammasome Activation through NEK7 Deglutathionylation. Cell Rep. 2019;29:151-161.e5.

82. Yang X, Li W, Zhang S, et al. PLK4 deubiquitination by Spata2-CYLD suppresses NEK7-mediated NLRP3 inflammasome activation at the centrosome. EMBO J. 2020;39(2):e102201.

83. Schmacke NA, Gaidt MM, Szymanska I, et al. Priming enables a NEK7independent route of NLRP3 activation. bioRxiv 799320; doi: https://doi.org/10.1101/799320

84. Chen Y, Meng J, Bi F, et al. NEK7 Regulates NLRP3 Inflammasome Activation and Neuroinflammation Post-traumatic Brain Injury. Front Mol Neurosci. 2019;12:202.

85. Ma ZZ, Sun HS, Lv JC, et al. Expression and clinical significance of the NEK7NLRP3 inflammasome signaling pathway in patients with systemic lupus erythematosus. J Inflamm (United Kingdom). 2018;15:16.

86. Chen X, Liu G, Yuan Y, et al. NEK7 interacts with NLRP3 to modulate the pyroptosis in inflammatory bowel disease via NF-kB signaling. Cell Death Dis. 2019;10:906.

87. Indramohan M, Stehlik C, Dorfleutner A. COPs and POPs Patrol Inflammasome Activation. J Mol Biol. 2018;430:153-173.

88. Matusiak M, Van Opdenbosch N, Lamkanfi M. CARD- and pyrin-only proteins regulating inflammasome activation and immunity. Immunol Rev. 2015;265:217-230.

89. Bedoya F, Sandler LL, Harton JA. Pyrin-Only Protein 2 Modulates NF-kB and Disrupts ASC:CLR Interactions. J Immunol. 2007;178:3837-3845.

90. Stehlik C, Krajewska M, Welsh K, et al. The PAAD/PYRIN-only protein POP1/ASC2 is a modulator of ASC-mediated nuclear-factor-kB and procaspase-1 regulation. Biochem J. 2003;373(Pt1):101-113.

91. Khare S, Ratsimandresy RA, De Almeida L, et al. The PYRIN domain-only protein POP3 inhibits ALR inflammasomes and regulates responses to infection with DNA viruses. Nat Immunol. 2014;15:343-353.

92. de Almeida L, Khare S, Misharin A V., et al. The PYRIN Domain-only Protein POP1 Inhibits Inflammasome Assembly and Ameliorates Inflammatory 
Disease. Immunity. 2015;43:264-276.

93. Periasamy S, Porter KA, Atianand MK, et al. Pyrin-only protein 2 limits inflammation but improves protection against bacteria. Nat Commun. 2017;8:15564.

94. Dorfleutner A, Bryan NB, Talbott SJ, et al. Cellular pyrin domain-only protein 2 is a candidate regulator of inflammasome activation. Infect Immun. 2007;75:1484-1492.

95. Ratsimandresy RA, Chu LH, Khare S, et al. The PYRIN domain-only protein POP2 inhibits inflammasome priming and activation. Nat Commun. 2017;8:15556.

96. Porter KA, Duffy EB, Nyland P, et al. The CLRX.1/NOD24 (NLRP2P) pseudogene codes a functional negative regulator of NF-KB, pyrin-only protein 4. Genes Immun. 2014;15:392-403.

97. Humke EW, Shriver SK, Starovasnik MA, et al. ICEBERG: A Novel Inhibitor of Interleukin-1 Generation. Cell. 2000;103:99-111.

98. Lamkanfi M, Denecker G, Kalai M, et al. INCA, a novel human caspase recruitment domain protein that inhibits interleukin-1 $\beta$ generation. J Biol Chem. 2004;279:51729-51738.

99. Druilhe A, Srinivasula SM, Razmara M, et al. Regulation of IL-1beta generation by Pseudo-ICE and ICEBERG, two dominant negative caspase recruitment domain proteins. Cell Death Differ. 2001;8:649-657.

100. Lee SH, Stehlik C, Reed JC. COP, a Caspase Recruitment Domain-containing Protein and Inhibitor of Caspase-1 Activation Processing. J Biol Chem. 2001;276:34495-34500.

101. Karasawa T, Kawashima A, Usui F, et al. Oligomerized CARD16 promotes caspase-1 assembly and IL-1 $\beta$ processing. FEBS Open Bio. 2015;5:348-356.

102. Lu A, Li Y, Schmidt FI, et al. Molecular basis of caspase-1 polymerization and its inhibition by a new capping mechanism. Nat Struct Mol Biol. 2016;23:416425.

103. Evans SS, Repasky EA, Fisher DT. Fever and the thermal regulation of immunity: The immune system feels the heat. Nat Rev Immunol. 2015;15:335349.

104. Carpenter S, Ricci EP, Mercier BC, et al. Post-transcriptional regulation of gene expression in innate immunity. Nat Rev Immunol. 2014;14:361-376.

105. Bryan NB, Dorfleutner A, Kramer SJ, et al. Differential splicing of the apoptosis-associated speck like protein containing a caspase recruitment domain (ASC) regulates inflammasomes. J Inflamm. 2010;7:23.

106. Cross R. One drug to cure them all. C\&EN Glob Enterp. 2020;98:26-31.

107. Mullard A. NLRP3 inhibitors stoke anti-inflammatory ambitions. Nat Rev Drug Discov. 2019;18:405-407.

108. Coll RC, Robertson AAB, Chae JJ, et al. A small-molecule inhibitor of the NLRP3 inflammasome for the treatment of inflammatory diseases. Nat Med. 2015;21:248-55.

109. Inflazome | Inzomelid completes Phase I studies and shows positive results in the treatment of Cryopyrin-Associated Periodic Syndrome (CAPS) Available from: http://www.inflazome.com/news/46. Accessed May 22, 2020.

110. Coll RC, Hill, Day JR, et al. MCC950 directly targets the NLRP3 ATPhydrolysis motif for inflammasome inhibition. Nat Chem Biol. 2019;15(6):556559.

111. Tapia-Abellán A, Angosto-Bazarra D, Martínez-Banaclocha H, et al. MCC950 
closes the active conformation of NLRP3 to an inactive state. Nat Chem Biol. 2019;15:560-564.

112. Gilmore TD, Herscovitch M. Inhibitors of NF-KB signaling: 785 and counting. Oncogene. 2006;25:6887-6899.

113. Herrington FD, Carmody RJ, Goodyear CS. Modulation of NF-kB Signaling as a Therapeutic Target in Autoimmunity. J Biomol Screen. 2016;21:223-242.

114. Juliana C, Fernandes-Alnemri T, Wu J, et al. Anti-inflammatory compounds parthenolide and bay 11-7082 are direct inhibitors of the inflammasome. J Biol Chem. 2010;285:9792-9802.

115. Robertson AAB. Inhibiting Inflammasomes with Small Molecules. Exp Suppl. 2018;108:343-400.

116. Krishnan N, Bencze $G$, Cohen $P$, et al. The anti-inflammatory compound BAY11-7082 is a potent inhibitor of protein tyrosine phosphatases. FEBS J. 2013;280:2830-2841.

117. Hausmann JS. Targeting cytokines to treat autoinflammatory diseases. Clin Immunol. 2019;206:23-32.

118. Dinarello CA. Biologic basis for interleukin-1 in disease. Blood. 1996;87:20952147.

119. Weber A, Wasiliew P, Kracht M. Interleukin-1 (IL-1) pathway. Science Signaling. 2010;3:cm1-cm1.

120. Franchi L, Eigenbrod T, Núñez G. Cutting Edge: TNF-a Mediates Sensitization to ATP and Silica via the NLRP3 Inflammasome in the Absence of Microbial Stimulation. J Immunol. 2009;183:792-796.

121. Tannahill GM, Curtis AM, Adamik J, et al. Succinate is an inflammatory signal that induces IL-1 $\beta$ through HIF-1 $\alpha$. Nature. 2013;496:238-42.

122. Hughes MM, O'Neill LAJ. Metabolic regulation of NLRP3. Immunol Rev. 2018;281:88-98.

123. Pålsson-McDermott EM, O'Neill LAJ. Targeting immunometabolism as an antiinflammatory strategy. Cell Research. 2020;30:300-314.

124. Wu Q, Wu W, Jacevic V, et al. Selective inhibitors for JNK signalling: a potential targeted therapy in cancer. J Enzyme Inhib Med Chem. 2020;35:574583.

125. Greenberg S, Horan G, Bennett B, et al. Evaluation of the JNK inhibitor, CC90001, in a phase 1b pulmonary fibrosis trial. Eur Respir J 2017; 50: Suppl. 61, OA5562.

126. Lee GS, Subramanian N, Kim Al, et al. The calcium-sensing receptor regulates the NLRP3 inflammasome through Ca2+ and cAMP. Nature. 2012;492:123127.

127. Sokolowska M, Chen L-Y, Liu Y, et al. Prostaglandin E 2 Inhibits NLRP3 Inflammasome Activation through EP4 Receptor and Intracellular Cyclic AMP in Human Macrophages . J Immunol. 2015;194:5472-5487.

128. Mullard A. Phosphatases start shedding their stigma of undruggability. Nat Rev Drug Discov. 2018;17:847-849.

129. O'Connor CM, Perl A, Leonard D, et al. Therapeutic targeting of PP2A. Int J Biochem Cell Biol. 2018;96:182-193.

130. Basiorka AA, Mcgraw KL, Eksioglu EA, et al. The NLRP3 inflammasome functions as a driver of the myelodysplastic syndrome phenotype. Blood. 2016;128(25):2960-2975.

131. Harrigan JA, Jacq X, Martin NM, et al. Deubiquitylating enzymes and drug discovery: Emerging opportunities. Nat Rev Drug Discov. 2018;17:57-77. 
132. Wertz IE, Wang X. From Discovery to Bedside: Targeting the Ubiquitin System. Cell Chem Biol. 2019;26:156-177.

133. Scott DE, Bayly AR, Abell C, et al. Small molecules, big targets: Drug discovery faces the protein-protein interaction challenge. Nat Rev Drug Discov. 2016;15:533-550.

134. Chen S, Dong D, Xin W, et al. Progress in the discovery of small molecule modulators of desumoylation. Curr Issues Mol Biol. 2020;35:17-34.

135. He X, Riceberg J, Soucy T, et al. Probing the roles of SUMOylation in cancer cell biology by using a selective SAE inhibitor. Nat Chem Biol. 2017;13:11641171.

136. Assouline S, Mehta A, Caimi PF, et al. A Phase 1b/2 Study of TAK-981, a First-in-Class Sumoylation Inhibitor, in Combination with Rituximab in Patients with Relapsed/Refractory (r/r) CD20-Positive Non-Hodgkin Lymphoma (NHL). Blood. 2019;134:1593-1593.

137. Dai $H$, Sinclair DA, Ellis JL, et al. Sirtuin activators and inhibitors: Promises, achievements, and challenges. Pharmacol Thera. 2018;188:140-154.

138. Dahlin JL, Nelson KM, Strasser JM, et al. Assay interference and off-target liabilities of reported histone acetyltransferase inhibitors. Nat Commun. 2017;8:1-14.

139. Byung HK, Roh E, Hwa YL, et al. Benzoxathiole derivative blocks lipopolysaccharide-induced nuclear factor-kB activation and nuclear factor-kBregulated gene transcription through inactivating inhibitory $\mathrm{kB}$ kinase $\beta$. Mol Pharmacol. 2008;73:1309-1318.

140. Bojadzic D, Buchwald P. Toward Small-Molecule Inhibition of Protein-Protein Interactions: General Aspects and Recent Progress in Targeting Costimulatory and Coinhibitory (Immune Checkpoint) Interactions. Curr Top Med Chem. 2018;18:674-699.

141. Bae JY, Park HH. Crystal structure of NALP3 Protein Pyrin Domain (PYD) and its implications in inflammasome assembly. J Biol Chem. 2011;286:3952839536.

142. Compositions and methods for modulation of immune response. C Stehlik - US Patent App. 15/117,629, 2016.

143. Desu HL, Plastini M, Illiano P, et al. IC100: a novel anti-ASC monoclonal antibody improves functional outcomes in an animal model of multiple sclerosis. J Neuroinflammation. 2020;17:143.

144. Netea MG, Nold-Petry CA, Nold MF, et al. Differential requirement for the activation of the inflammasome for processing and release of IL-1 $\beta$ in monocytes and macrophages. Blood. 2009;113:2324-2335.

145. Gritsenko A, Yu S, Martin-Sanchez F, et al. Priming is dispensable for NLRP3 inflammasome activation in human monocytes. bioRxiv 2020.01.30.925248; doi: https://doi.org/10.1101/2020.01.30.925248.

146. Hoss F, Mueller JL, Rojas Ringeling F, et al. Alternative splicing regulates stochastic NLRP3 activity. Nat Commun. 2019;10:1-13.

147. Schroder K, Irvine KM, Taylor MS, et al. Conservation and divergence in Tolllike receptor 4-regulated gene expression in primary human versus mouse macrophages. Proc Natl Acad Sci U S A. 2012 Apr 17;109(16):E944-53.

148. Kapetanovic R., Ariffin J.K., Sweet M.J. (2014) Evolutionary Divergence in Human Versus Mouse Innate Immune Gene Regulation and Function. In: Pontarotti P. (eds) Evolutionary Biology: Genome Evolution, Speciation, Coevolution and Origin of Life. Springer, Cham DOI: 10.1007/978-3-319- 
07623-2_6,

149. Coll R, O'Neill L, Schroder K. Questions and controversies in innate immune research: what is the physiological role of NLRP3? Cell Death Discovery. $2016 ; 2: 1-5$.

Figures 


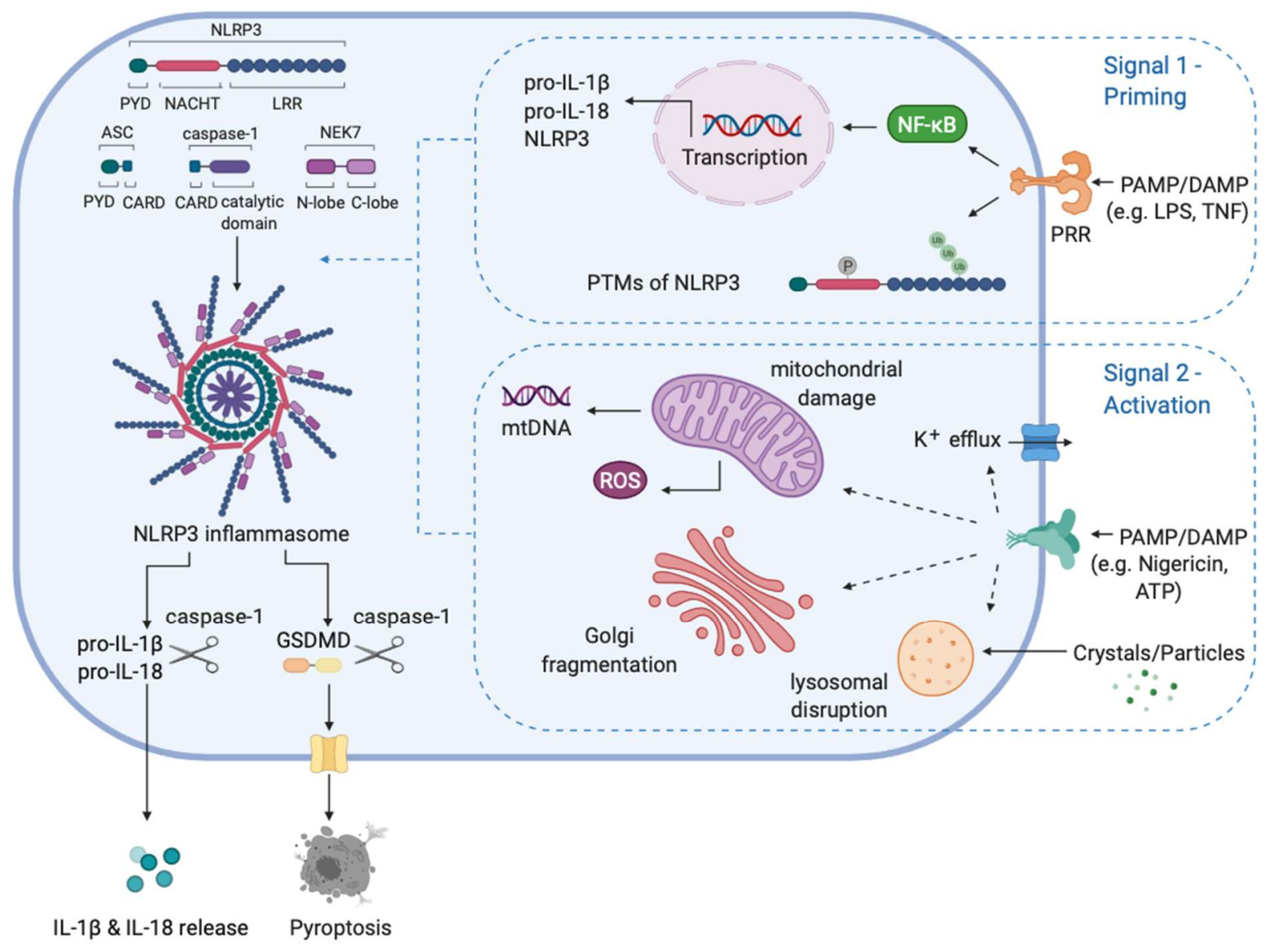

Figure 1; NLRP3 inflammasome activation. Priming involves binding of PAMPs and DAMPs, such as LPS and TNF, to pattern recognition receptors (PRRs) triggering upregulation of NLRP3, pro-IL-1 $\beta$ and pro-IL-18 transcription via NF-KBdependent pathways. Priming also triggers PTMs of NLRP3 to facilitate rapid NLRP3 regulation. PAMPs and DAMPs, such as nigericin or extracellular ATP, or crystals/particles act as the second activating signal. This triggers various intracellular events, including $\mathrm{K}+$ efflux, lysosomal disruption, dispersal of the transGolgi network and mitochondrial damage leading to release of mitochondrial (mt)DNA and production of ROS. Together, priming and activation stimuli induce NLRP3 oligomerisation which recruits ASC, triggering formation of the ASC speck and recruiting pro-caspase-1. The inflammasome complex also recruits NEK7 which mediates formation of interactions between adjacent NLRP3 subunits. Pro-caspase- 
1 auto-catalytically self-cleaves to become active and then cleaves its effector substrates, pro-IL-1 $\beta$, pro-IL-18 and GSDMD. Mature forms of these cytokines are released from the cell and cleaved GSDMD forms pores in the plasma membrane, triggering pyroptosis.

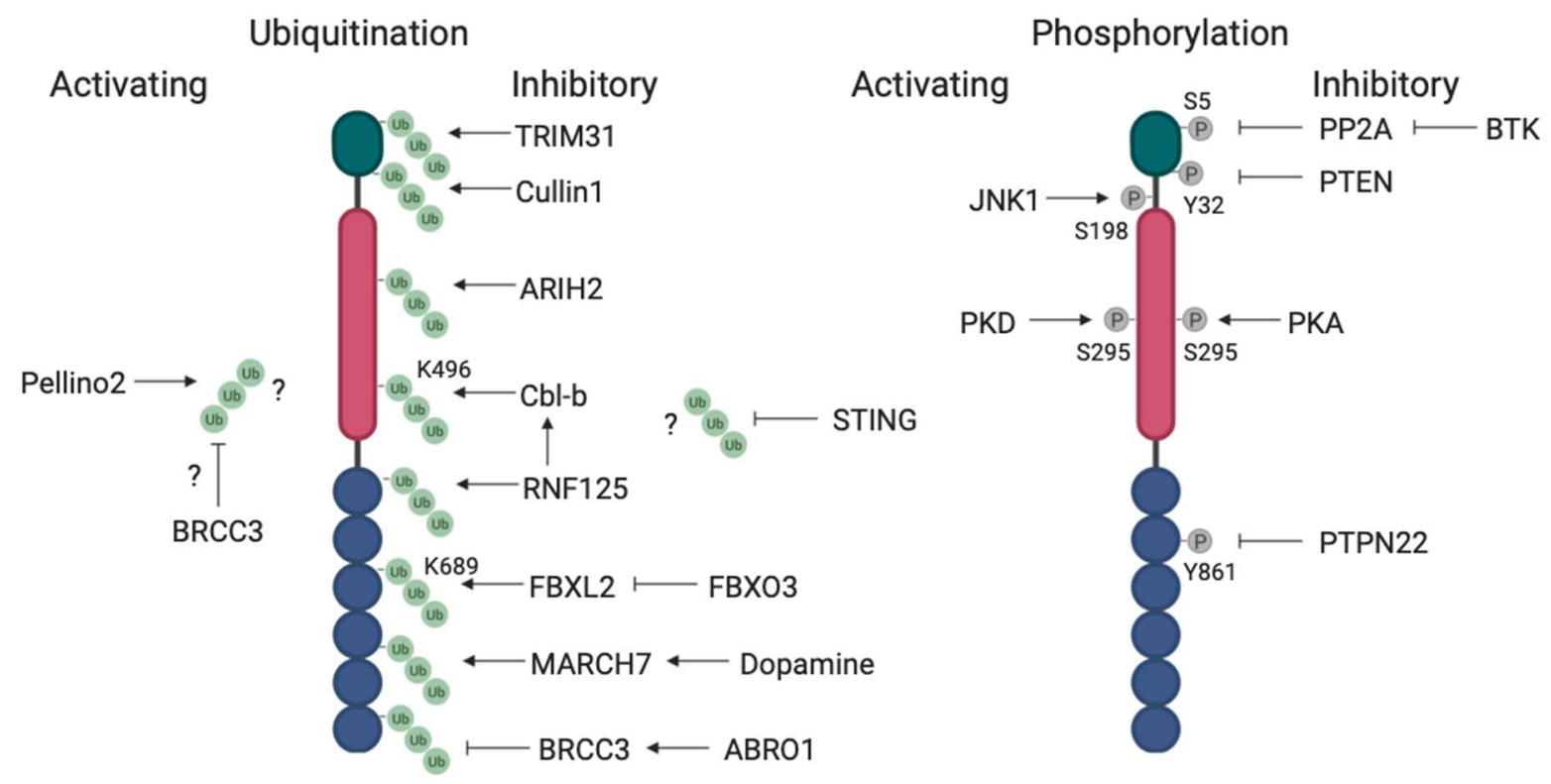

Sumoylation
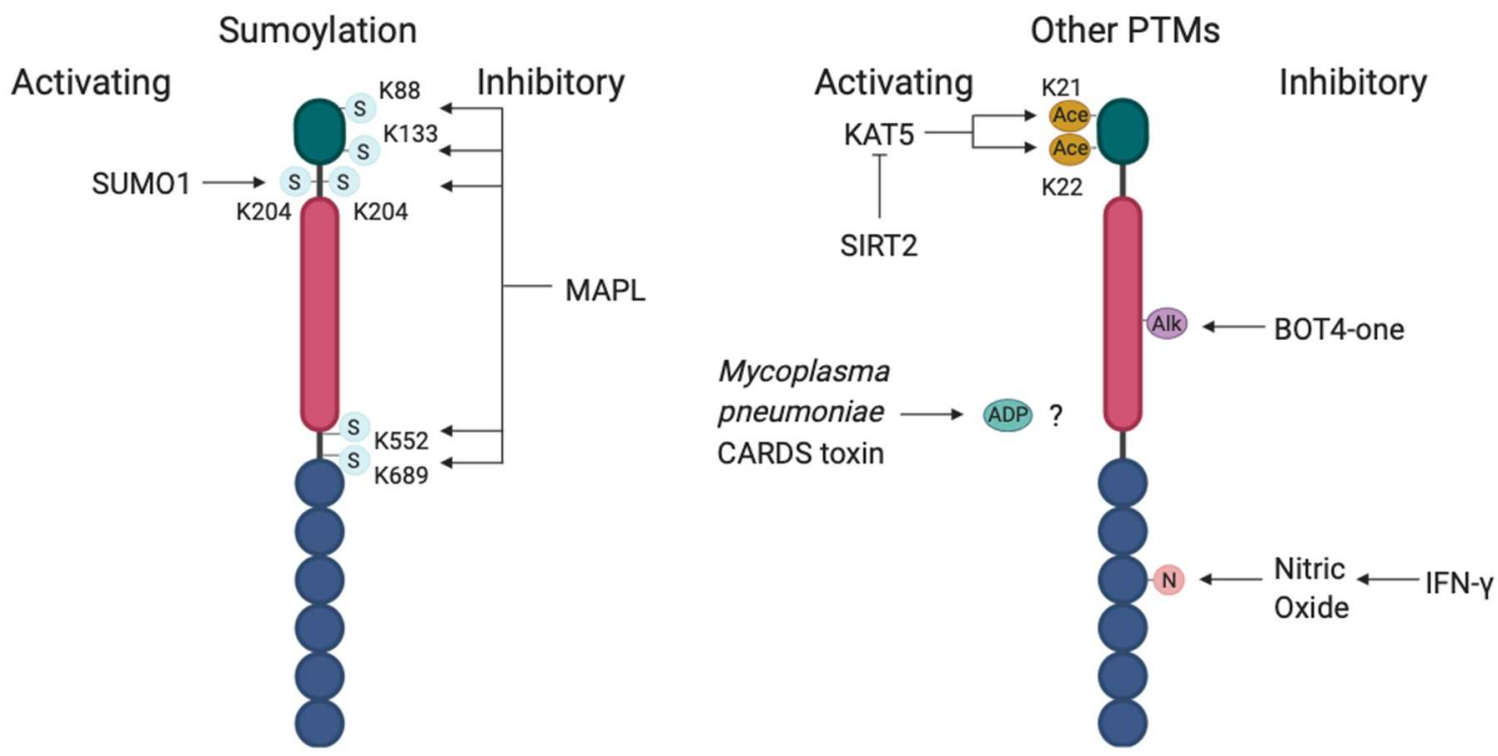
Figure 2; PTMs of NLRP3. Activation of NLRP3 is regulated via PTMs including ubiquitination $(\mathrm{Ub})$, phosphorylation $(\mathrm{P})$, sumoylation $(\mathrm{S})$, nitrosylation $(\mathrm{N})$, acetylation (Ace), alkylation (Alk) and ADP-risobylation (ADP). These PTMs can both activate (left) and inhibit (right) the NLRP3 inflammasome. Specific amino acid sites of modification are included where known.

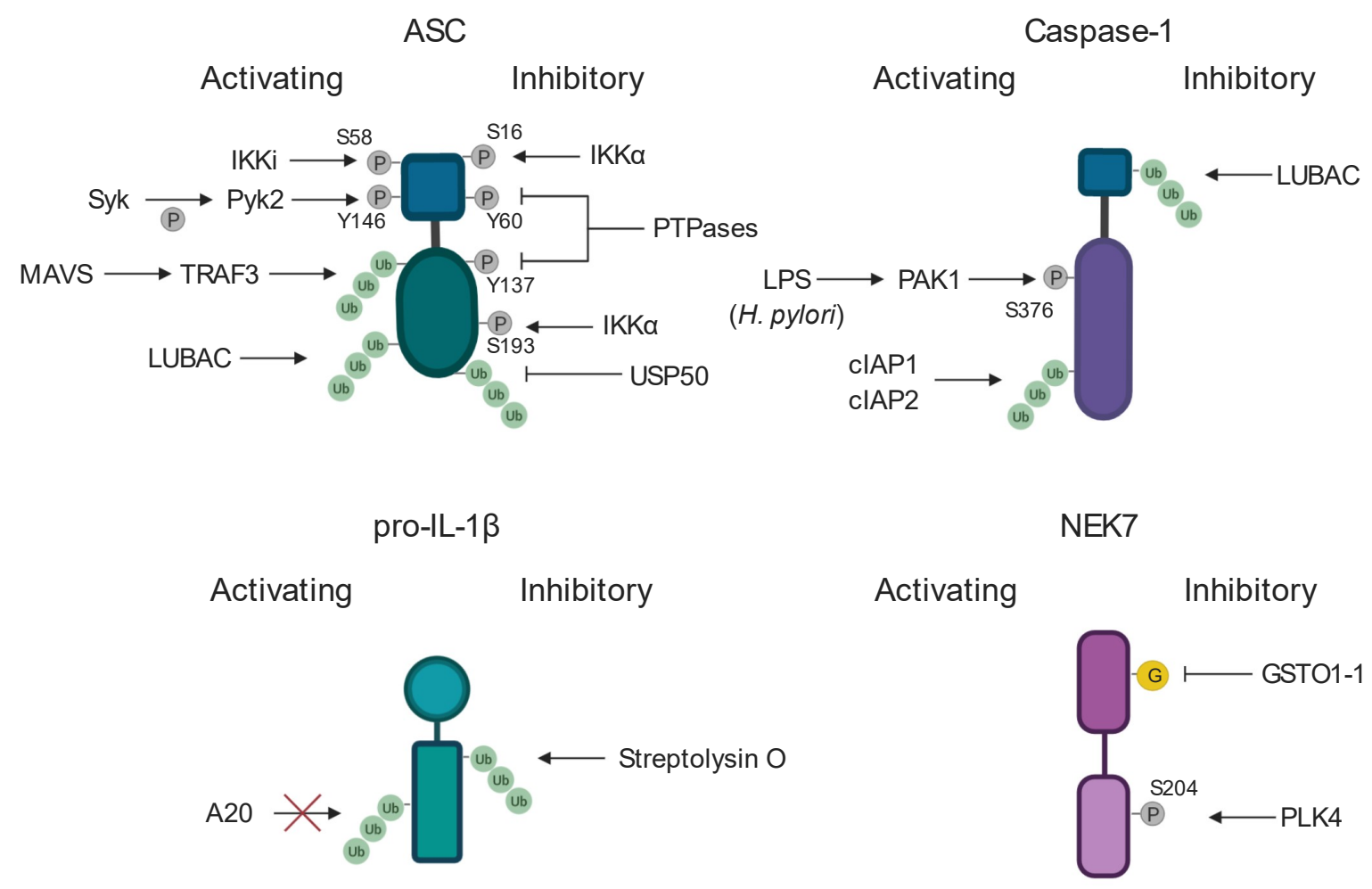

Figure 3; PTMs of additional inflammasome components. NLRP3 inflammasome formation is regulated via PTMs of the inflammasome components ASC, caspase-1, pro-IL-1 $\beta$ and NEK7. These PTMs include ubiquitination (Ub), phosphorylation (P) and glutathionylation $(\mathrm{G})$, which can both activate (left) and inhibit (right) NLRP3 inflammasome signalling. Specific amino acid sites of modification are included where known. 


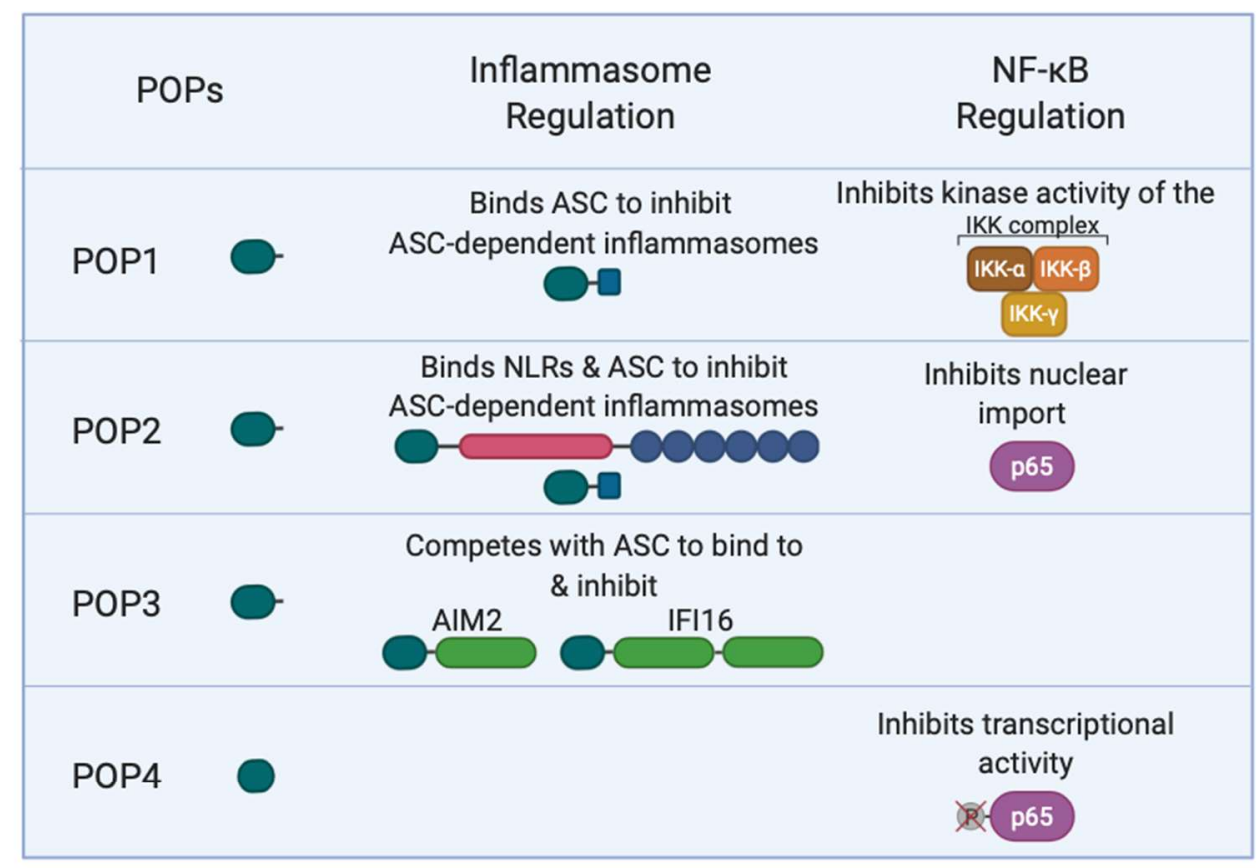

\begin{tabular}{|ccc|c|}
\hline COPs & $\begin{array}{c}\text { Caspase-1 } \\
\text { Regulation }\end{array}$ \\
\hline CARD17 & - & Prevents CARD-CARD \\
interactions \\
Oligomerised CARD16 \\
CARD18
\end{tabular}

Figure 4; Roles of POPs and COPs in regulating inflammation. A summary of the roles of POPs in regulating activation of inflammasomes and NF-KB and the roles of COPs in regulating activation of caspase- 1 . 


\begin{tabular}{|c|c|c|}
\hline \multicolumn{3}{|c|}{ Direct NLRP3 inhibition } \\
\hline Target & Inhibitor & Effect \\
\hline $\begin{array}{l}\text { NLRP3 } \\
\text { Walker B }\end{array}$ & $\begin{array}{c}\text { MCC950 } \\
\text { (aka CP-456,773) }\end{array}$ & $\begin{array}{l}\text { ATPase } \\
\text { activity }\end{array}$ \\
\hline \multicolumn{3}{|c|}{ Transcriptional } \\
\hline Target & Inhibitor & Effect \\
\hline$N F-k B$ & $\begin{array}{l}\text { 100s of molecules } \\
\text { e.g. BAY 11-7082 }\end{array}$ & $\begin{array}{l}\text { pro-IL-1 } \beta \\
\text { NLRP3 }\end{array}$ \\
\hline Glycolysis & 2-deoxyglucose & $\downarrow$ pro-lL-1 $\beta$ \\
\hline \multicolumn{3}{|c|}{ Phosphorylation } \\
\hline Target & Inhibitor/activator & Effect \\
\hline JNK1 & SP600125, CC-90001 & $\downarrow$ (P) $\mathrm{S} 198$ \\
\hline PKA & $\begin{array}{c}\text { dibutyryl cAMP, } \\
\text { phosphodiesterase inhibitors }\end{array}$ & $\uparrow$ (P) S295 \\
\hline PKD & CRT 0066101, kb NB 142-70 & $\downarrow$ (P) $\mathrm{S} 295$ \\
\hline PKD & Okadaic acid, LB-100 & $\uparrow(P) S 5$ \\
\hline
\end{tabular}

\begin{tabular}{|c|c|c|}
\hline \multicolumn{3}{|c|}{ Ubiquitination } \\
\hline Target & Inhibitor/activator & Effect \\
\hline (BRCC3) & G5 & $\uparrow \cup b \cup b \cup b$ \\
\hline USP7) & P22077, HBX19818 & $\uparrow U b \quad U b, U b$ \\
\hline \multicolumn{3}{|c|}{ Additional PTMs } \\
\hline Target & Inhibitor/activator & Effect \\
\hline SUMO) & Tak-981 & $\downarrow$ (S) K204 \\
\hline Kat 5 & NU9056 & $\downarrow$ Ace $\mathrm{K} 21, \mathrm{~K} 22$ \\
\hline Cys residues & Bot-4-one & ALK Cys \\
\hline
\end{tabular}

\begin{tabular}{|c|}
\hline \multicolumn{2}{|c|}{ Protein binding partners } \\
\hline Type of molecule
\end{tabular}

Figure 5; Potential targets involved in NLRP3 priming, PTMs, and PPIs for antiinflammatory therapies. Direct inhibitors of NLRP3 such as MCC950 are priming independent. A summary of potential targets and therapeutic strategies affecting NLRP3 transcriptional priming, NLRP3 post-translational modifications (phosphorylation, ubiquitination, acetylation, sumoylation, and alkylation), and PPIs that influence NLRP3 inflammasome formation. Some examples of currently available drugs and molecules are included. 